\title{
Bayesian Analysis of the Mixture of Frechet Distribution under Different Loss Functions
}

\author{
Tabasam Sultana \\ Department of Statistics, Quaid-i-Azam University, Islamabad, Pakistan \\ tabasamsultana08@gmail.com
}

Muhammad Aslam

A Department of Basic Sciences, Ripha International University, Islamabad, 44000, Pakistan

aslamsdqu@yahoo.com.

Javid Shabbir

Department of Statistics, Quaid-i-Azam University, Islamabad, Pakistan

javidshabbir@gmail.com

\begin{abstract}
This paper has to do with 3-component mixture of the Frechet distributions when the shape parameter is known under Bayesian view point. The type-I right censored sampling scheme is considered due to its extensive use in reliability theory and survival analysis. Taking different non-informative and informative priors, Bayes estimates of the parameter of the mixture model along with their posterior risks are derived under squared error loss function, precautionary loss function and DeGroot loss function. In case, no or little prior information is available, elicitation of hyper parameters is given. In order to study numerically, the execution of the Bayes estimators under different loss functions, their statistical properties have been simulated for different sample sizes and test termination times. A real life data example is also given to illustrate the study.
\end{abstract}

Keywords: Bayes Estimators, Censoring, Informative prior, Loss Functions, Posterior Risks.

\section{Introduction}

Frechet distribution was introduced by a French mathematician named Maurice Frechet $(1878,1973)$ who had determined before one possible limit distribution for the largest order statistic in 1927. The Frechet distribution has been manifested to be helpful for modeling and analysis of several extreme events ranging from accelerated life testing to earthquakes, floods, rain fall, sea currents and wind speed.

Applications of the Frechet distribution in many fields given in Harlow (2002) showed that it is an important distribution for modeling the statistical behavior of materials properties for a variety of engineering implementation. In hydrology, the Frechet distribution is applied to extreme events such as annually maximum one day rainfalls and river discharges. Nadarajah and Kotz (2008) described the sociological models based on Frechet random variables. Zaharim et al. (2009) applied Frechet distribution for analyzing the wind speed data. Chatterjee and Chatterjee (2012) used Frechet distribution to measure ultrasonic pulse. Abbas et al. (2012) described the comparison methods for Frechet distribution with known shape. 
Anwar et al. (2014) used Frechet distribution to study analysis of accelerated life testing by using Geometric process. Abbas et al. (2015) discussed the analysis of Frechet distribution using the reference priors. The Bayesian and Maximum likelihood estimators are compared via simulation study.

Several types of data are encountered in everyday life, regarding simple data, grouped data, truncated data, censored data and progressively censored data. Censoring is an inevitable part of the lifetime data. A valuable account of censoring is given in Gijbles (2010) and Kalbfleisch and Prentice (2011). There are different sorts of censoring schemes, including right, left and interval censoring, single or multiple censoring and type-1 and type-II censoring. Kundu and Howlader (2010) discussed the Bayesian inference and prediction of the IW distribution for type-II censored data. Shi and Yan (2010) derived the empirical Bayes estimates of the two parameter exponential distribution under type-I censoring. Saleem et al. (2010) discussed Bayesian analysis on the power function mixture distribution using typeI censored data. Ali (2015) described the 2-component mixture of the inverse Rayleigh distributions under Bayesian framework. Aslam et al. (2015) presented 3-component mixture of Rayleigh distributions, properties and estimation under the Bayesian framework.

Inspired by above mentioned applications of mixture models, we intend to study Bayesian analysis of a 3-component mixture of the Frechet distributions with unknown mixing proportions. The parameters of component distributions are assumed to be unknown. Four different priors and three different loss functions are used for the Bayesian analysis. Moreover, we consider an ordinary type-I right censored sampling schemes.

The structure of this article is as follows. The Frechet mixture model along with its likelihood function is formulated in section 2. The expressions for posterior distributions using the non-informative and informative priors are derived in section 3 . In section 4, the Bayes estimators and posterior risks using the uniform the J effreys', the exponential and the inverse levy priors under squared error loss function (SELF), precautionary loss function (PLF) and DeGroot loss function (DLF) are presented. The elicitation of hyperparameters is given in section 5. In section 6, the limiting expressions of the Bayes estimators and their posterior risks are derived. The simulation study and the real data applications are presented in section 7 and 8, respectively. This article concludes with a brief discussion in section 9 .

\section{3-Component mixture of the Frechet distributions}

The probability density function (p.d.f) and the cumulative distribution function (c.d.f) of the Frechet distribution for a random variable $\mathrm{X}$ are given by:

$$
f(x ; \alpha, \beta)=\frac{\alpha}{\beta}\left(\frac{\beta}{x}\right)^{\alpha+1} \exp \left[-\left(\frac{\beta}{x}\right)^{\alpha}\right], x>0
$$

Where the parameter $\alpha>0$ determines the shape of the distribution and $\beta>0$ is the scale parameter.

$$
F(x)=\exp \left[-\left(\frac{\beta}{x}\right)^{\alpha}\right], x>0
$$


When the shape parameter $\alpha=1$, then the above p.d.f and c.d.f will become as:

$$
\begin{aligned}
& f_{m}\left(x ; \beta_{m}\right)=\left(\frac{\beta_{m}}{x^{2}}\right) \exp \left[-\left(\frac{\beta_{m}}{x}\right)\right] ; x \geq 0, \beta_{m}>0, m=1,2,3 \\
& F_{m}(x)=\exp \left[-\left(\frac{\beta_{m}}{x}\right)\right]
\end{aligned}
$$

A finite 3-component mixture model with the unknown mixing proportions $p_{1}$ and $p_{2}$ is:

$$
\begin{array}{r}
f(x)=p_{1} f_{1}(x)+p_{2} f_{2}(x)+\left(1-p_{1}-p_{2}\right) f_{3}(x), p_{1}, p_{2} \geq 0, p_{1}+p_{2} \leq 1 \\
f\left(x, \beta_{1}, \beta_{2}, \beta_{3}, p_{1}, p_{2}\right)=p_{1}\left(\frac{\beta_{1}}{x^{2}}\right) \exp \left[-\left(\frac{\beta_{1}}{x}\right)\right]+p_{2}\left(\frac{\beta_{2}}{x^{2}}\right) \exp \left[-\left(\frac{\beta_{2}}{x}\right)\right] \\
+\left(1-p_{1}-p_{2}\right)\left(\frac{\beta_{3}}{x^{2}}\right) \exp \left[-\left(\frac{\beta_{2}}{x}\right)\right] ; p_{1}, p_{2} \geq 0, p_{1}+p_{2} \leq 1
\end{array}
$$

While the c.d.f of 3-component mixture model is:

$$
\begin{aligned}
& F(x)=p_{1} F_{1}(x)+p_{2} F_{2}(x)+\left(1-p_{1}-p_{2}\right) F_{3}(x) \\
& F(x)=p_{1} \exp \left[-\left(\frac{\beta_{1}}{x}\right)\right]+p_{2} \exp \left[-\left(\frac{\beta_{2}}{x}\right)\right]+\left(1-p_{1}-p_{2}\right) \exp \left[-\left(\frac{\beta_{3}}{x}\right)\right]
\end{aligned}
$$

2.1. The Likelihood Function. Suppose ' $n$ ' units from the 3 -component mixture of Frechet distributions are used in a life testing experiment with fixed test termination time $t$. Let ' $r$ ' units out of ' $n$ ' units failed until fixed test termination time ' $t$ ' and the remaining (n-r) units are still working. According to Mendenhall and Hader (1958), there are many practical situations in which the failed objects can be pointed out easily as subset of subpopulation-I, subpopulation-II or subpopulation-III. Out of ' $r$ ' units, supposer $r_{1}, r_{2}$ and $r_{3}$ units belong to subpopulation-I, subpopulation-II or subpopulation-III respectively and such that $r=r_{1}+r_{2}+r_{3}$. Now we define $x_{l k}, 0<x_{l k}<t$ be the failure time of $k^{\text {th }}$ unit belonging to the $l^{\text {th }}$ subpopulation, where $l=1,2,3$ and $k=1,2, \ldots, r_{l}$. For a 3-component mixture model, the likelihood function can be written as:

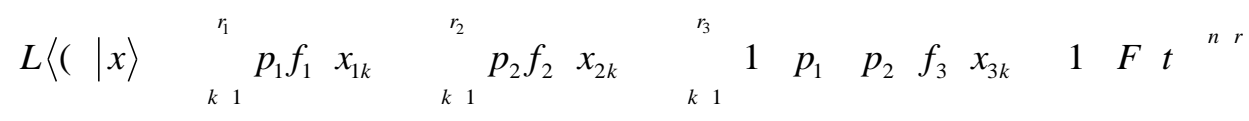

After simplification, the likelihood function of 3-component mixture of Frechet distributions is given:

$$
\begin{aligned}
L(\phi \mid \mathbf{x}) & \propto \beta_{1}^{r_{1}} \beta_{2}^{r_{2}} \beta_{3}^{r_{3}} \sum_{i=0}^{n-r} \sum_{j=0}^{i} \sum_{l=0}^{j}(-1)^{i}\left(\begin{array}{c}
n-r \\
i
\end{array}\right)\left(\begin{array}{c}
i \\
j
\end{array}\right)\left(\begin{array}{c}
j \\
l
\end{array}\right) \\
& \times \exp \left\{-\beta_{1}\left(\sum_{k=1}^{r_{1}} x_{1 k}^{-1}+\frac{i-j}{t}\right)\right\} \exp \left\{-\beta_{2}\left(\sum_{k=1}^{r_{2}} x_{2 k}^{-1}+\frac{j-l}{t}\right)\right\} \\
& \times \exp \left\{-\beta_{3}\left(\sum_{k=1}^{r_{3}} x_{3 k}^{-1}+\frac{l}{t}\right)\right\} p_{1}^{i-j+r_{1}} p_{2}^{j-l+r_{2}}\left(1-p_{1}-p_{2}\right)^{l+r_{3}}
\end{aligned}
$$

Where $\mathbf{X}=\left(x_{1}, x_{12}, \ldots, x_{1 r 1}, x_{21}, x_{22}, \ldots, x_{2 r 2}, x_{31}, x_{32}, \ldots, x_{3 r 3}\right)$ are the observed failure times for the uncensored observations and $\square \square \square \square_{1}, \square_{2}, \square_{3}, p_{1}, p_{2}[$. 
3. The posterior distribution using the non-informative and the informative priors

In this section, posterior distributions of parameters given data, say $\mathrm{x}$, are derived using the non-informative (Uniform and Jeffreys') and the informative (Exponential and Inverse Levy) priors.

3.1. The posterior distribution using the Uniform Prior (UP). When elicitation of hyper parameters is difficult or little prior information is given, then usually the noninformative prior is assumed to be the UP. Ups over the intervals $(0, \infty)$ and $(0,1)$ are taken for the parameters $\left(\beta_{1}, \beta_{2} \& \beta_{3}\right)$ of Frechet distribution and for the mixing proportions $\left(p_{1}, p_{2}\right)$ respectively. With these settings, joint prior distribution of parameters $\left(\beta_{1}, \beta_{2}, \beta_{3}, p_{1}, p_{2}\right)$, is given by:

\section{$\pi(\varphi) \propto 1 ; \beta_{1}, \beta_{2}, \beta_{3}>0, p_{1}, p_{2} \geq 0, p_{1}+p_{2} \leq 1$}

The joint posterior distribution of parameters $\beta_{1}, \beta_{2}, \beta_{3}, p_{1}$ and $p_{2}$ given data $\mathbf{x}$ assuming the UP is:

$$
\begin{aligned}
g_{1}(\phi \mid \mathbf{x}) & =\frac{L\langle\phi \mid \mathbf{x}\rangle \pi_{1}(\phi)}{\int_{\phi} L\langle\phi \mid \mathbf{x}\rangle \pi_{1}(\phi) d \phi} \\
g_{1}(\phi \mid \mathbf{x})= & C_{1}^{-1} \sum_{i=0}^{n-r} \sum_{j=0}^{i} \sum_{l=0}^{j}(-1)^{i}\left(\begin{array}{c}
n-r \\
i
\end{array}\right)\left(\begin{array}{c}
i \\
j
\end{array}\right)\left(\begin{array}{c}
j \\
l
\end{array}\right) \\
& \times \beta_{1}^{A_{11}-1} \beta_{2}^{A_{21}-1} \beta_{3}^{A_{31}-1} \exp \left(-\beta_{1} M_{11}\right) \exp \left(-\beta_{2} M_{21}\right) \\
& \times \exp \left(-\beta_{3} M_{31}\right) p_{1}^{A_{01}-1} p_{2}^{B_{01}-1}\left(1-p_{1}-p_{2}\right)^{C_{01}-1}
\end{aligned}
$$

where

$$
\begin{aligned}
& A_{11} \square r_{1} \square 1, A_{21} \square r_{2} \square 1, A_{31} \square r_{3} \square 1, M_{11} \square \underset{k \square 1}{r_{1}^{\prime}} x_{1 k}^{\square 1} \square \frac{i \square j}{t}, M_{21} \square \underset{k \square 1}{\square} x_{2 k}^{r_{2}} \square \frac{j \square l}{t}, \\
& M_{31} \square \underset{k \square 1}{r_{3}^{\square}} x_{3 k}^{\square 1} \square \frac{l}{t}, A_{01} \square i \square j \square r_{1} \square 1, B_{01} \square j \square l \square r_{2} \square 1, C_{01} \square l \square r_{3} \square 1,
\end{aligned}
$$

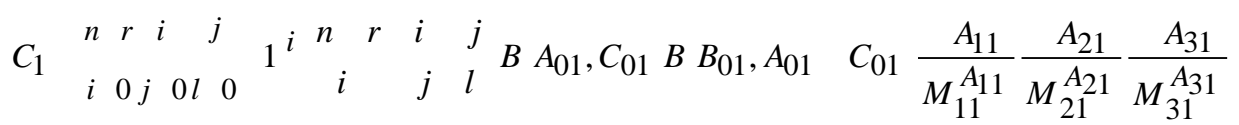

\subsection{Theposterior distribution using the J effreys' prior ( $\mathrm{P})$.}

According to Jeffreys' (1946, 1998) and Berger (1985), the JP is defined as

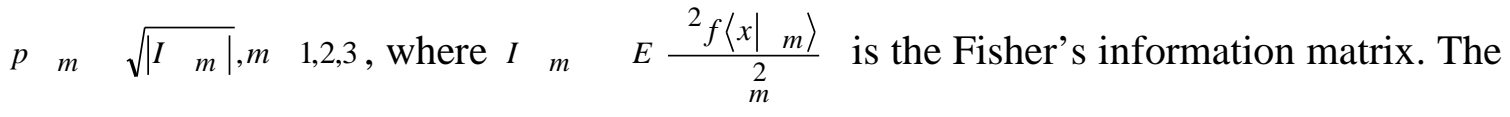

prior distributions of the mixing proportions $p_{1}$ and $p_{2}$ are again taken to be the uniform over the interval $\emptyset, 1[$. Under the assumption of independence of all parameters, the joint prior distribution of $\square_{1}, \square_{2}, \square_{3}, p_{1}, p_{2}[$ is:

$$
\pi_{2}(\phi) \propto \frac{1}{\beta_{1} \beta_{2} \beta_{3}}, \beta_{1}, \beta_{2}, \beta_{3} \geq 0, p_{1}, p_{2} \geq 0, p_{1}+p_{2} \leq 1
$$


The joint posterior distribution of parameters $\beta_{1}, \beta_{2}, \beta_{3}, p_{1}$ and $p_{2}$ given data $\mathbf{x}$ assuming the JP is:

$$
\begin{aligned}
g_{2}\langle\phi \mid \mathbf{x}\rangle & =\frac{L\langle\phi \mid \mathbf{x}\rangle \pi_{2}(\phi)}{\int_{\phi} L\langle\phi \mid \mathbf{x}\rangle \pi_{2}(\phi) d \phi} \\
g_{2}(\phi \mid \mathbf{x})= & C_{2}^{-1} \sum_{i=0}^{n-r} \sum_{j=0}^{i} \sum_{l=0}^{j}(-1)^{i}\left(\begin{array}{c}
n-r \\
i
\end{array}\right)\left(\begin{array}{c}
i \\
j
\end{array}\right)\left(\begin{array}{c}
j \\
l
\end{array}\right) \\
& \times \beta_{1}^{A_{12}-1} \beta_{2}^{A_{22}-1} \beta_{3}^{A_{32}-1} \exp \left(-\beta_{1} M_{12}\right) \exp \left(-\beta_{2} M_{22}\right) \\
& \times \exp \left(-\beta_{3} M_{32}\right) p_{1}^{A_{02}-1} p_{2}^{B_{02}-1}\left(1-p_{1}-p_{2}\right)^{C_{02}-1}
\end{aligned}
$$

where

$$
\begin{aligned}
& A_{12} \square r_{1}, A_{22} \square r_{2}, A_{32} \square r_{3}, M_{12} \square \underset{k \square 1}{\stackrel{r_{1}}{\square}} x_{1 k}^{\square 1} \square \frac{i \square j}{t}, M_{22} \square \underset{k \square 1}{\stackrel{r_{2}}{\square}} x_{2 k}^{\square 1} \square \frac{j \square l}{t}, \\
& M_{32 \square} \underset{k \square 1}{r_{3}^{3}} x_{3 k}^{\square 1} \square \frac{l}{t}, A_{02} \square i \square j \square r_{1} \square 1, B_{02} \square j \square l \square r_{2} \square 1, C_{02} \square l \square r_{3} \square 1,
\end{aligned}
$$

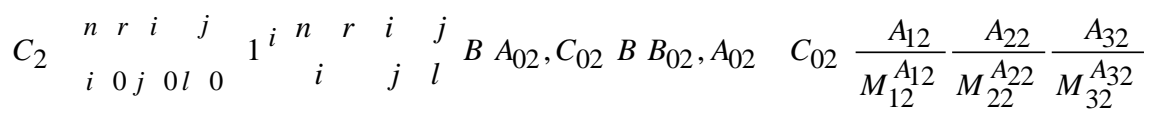

3.3. The posterior distribution using the Exponential prior (EP). As an informative prior, we take the exponential prior for the component parameters $\beta_{1}, \beta_{2}, \beta_{3}$ and Bivariate Beta prior for proportion parameters $p_{1}, p_{2}$. Symbolically, it can be written as: $\beta_{1} \sim$ Exponential $\left(k_{1}\right), \beta_{2} \sim \operatorname{Exponential}\left(k_{2}\right), \beta_{3} \sim \operatorname{Exponential}\left(k_{3}\right)$ and $p_{1}, p_{2} \sim \operatorname{Bivariate} \operatorname{Beta}(a$, $b, c)$. Again assuming independence of all parameters, the joint prior distribution of $\left(\beta_{1}\right.$, $\left.\beta_{2}, \beta_{3}, p_{1}, p_{2}\right)$ is given by:

$$
\pi_{3}(\phi) \propto \exp \left(-\beta_{1} k_{1}\right) \exp \left(-\beta_{2} k_{2}\right) \exp \left(-\beta_{3} k_{3}\right) p_{1}^{a-1} p_{2}^{b-1}\left(1-p_{1}-p_{2}\right)^{c-1}
$$

The joint posterior distribution of parameters $\beta_{1}, \beta_{2}, \beta_{3}, p_{1}$ and $p_{2}$ given data $\mathbf{x}$ is:

$$
\begin{aligned}
g_{3}\langle\phi \mid \mathbf{x}\rangle= & \frac{L\langle\phi \mid \mathbf{x}\rangle \pi_{3}(\phi)}{\int_{\phi} L\langle\phi \mid \mathbf{x}\rangle \pi_{3}(\phi) d \phi} \\
g_{3}(\phi \mid \mathbf{x})= & C_{3}^{-1} \sum_{i=0}^{n-r} \sum_{j=0}^{i} \sum_{l=0}^{j}(-1)^{i}\left(\begin{array}{c}
n-r \\
i
\end{array}\right)\left(\begin{array}{c}
i \\
j
\end{array}\right)\left(\begin{array}{c}
j \\
l
\end{array}\right) \\
& \times \beta_{1}^{A_{13}-1} \beta_{2}^{A_{23}-1} \beta_{3}^{A_{33}-1} \exp \left(-\beta_{1} M_{13}\right) \exp \left(-\beta_{2} M_{23}\right) \\
& \times \exp \left(-\beta_{3} M_{33}\right) p_{1}^{A_{03}-1} p_{2}^{B_{03}-1}\left(1-p_{1}-p_{2}\right)^{C_{03}-1}
\end{aligned}
$$

where

$$
\begin{aligned}
& A_{13} \square r_{1} \square 1, A_{23} \square r_{2} \square 1, A_{32} \square r_{3} \square 1, M_{13} \square \underset{k \square 1}{\prod_{d}^{n}} x_{1 k}^{\square 1} \square \frac{i \square j}{t} \square k_{1}, M_{23} \square \underset{k \square 1}{r_{2}^{2}} x_{2 k}^{\square 1} \square \frac{j \square l}{t} \square k_{2}, \\
& M_{33} \square \underset{k \square 1}{r_{3}} x_{3 k}^{\square 1} \square \frac{l}{t} \square k_{3}, A_{03} \square i \square j \square r_{1} \square a, B_{03} \square j \square l \square r_{2} \square b, C_{02} \square l \square r_{3} \square c,
\end{aligned}
$$

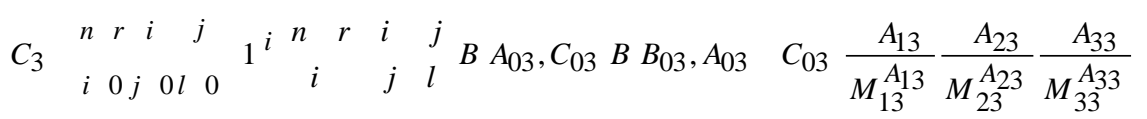


3.4. The posterior distribution using the Inverse Levy prior (ILP). As an informative prior, we take the Inverse Levy prior for the component parameters $\beta_{1}, \beta_{2}, \beta_{3}$ and Bivariate Beta prior for proportion parameters $p_{1}, p_{2}$. Symbolically, it can be written as: $\beta_{1} \sim$ Inverselevy $\left(a_{1}\right), \beta_{2} \sim$ Inverselevy $\left(a_{2}\right), \beta_{3} \sim$ Inverselevy $\left(a_{3}\right)$ and $p_{1}, p_{2} \sim$ Bivariate Beta $(a, b, c)$. Again assuming independence of all parameters, the joint prior distribution of $\left(\beta_{1}, \beta_{2}, \beta_{3}, p_{1}, p_{2}\right)$ is given by:

$$
\pi_{4}(\phi) \propto \beta_{1}^{\frac{-1}{2}} \exp \left(-\frac{a_{1} \beta_{1}}{2}\right) \beta_{2}^{\frac{-1}{2}} \exp \left(-\frac{a_{2} \beta_{2}}{2}\right) \beta_{3}^{\frac{-1}{2}} \exp \left(-\frac{a_{3} \beta_{3}}{2}\right) p_{1}^{a-1} p_{2}^{b-1}\left(1-p_{1}-p_{2}\right)^{c-1}
$$

The joint posterior distribution of parameters $\beta_{1}, \beta_{2}, \beta_{3}, p_{1}$ and $p_{2}$ given data $\mathbf{x}$ is:

$$
\begin{aligned}
g_{4}\langle\phi \mid \mathbf{x}\rangle= & \frac{L\langle\phi \mid \mathbf{x}\rangle \pi_{4}(\phi)}{\int_{\phi} L\langle\phi \mid \mathbf{x}\rangle \pi_{4}(\phi) d \phi} \\
g_{4}(\phi \mid \mathbf{x})= & C_{4}^{-1} \sum_{i=0}^{n-r} \sum_{j=0}^{i} \sum_{l=0}^{j}(-1)^{i}\left(\begin{array}{c}
n-r \\
i
\end{array}\right)\left(\begin{array}{c}
i \\
j
\end{array}\right)\left(\begin{array}{c}
j \\
l
\end{array}\right) \\
& \times \beta_{1}^{A_{14}-1} \beta_{2}^{A_{24}-1} \beta_{3}^{A_{34}-1} \exp \left(-\beta_{1} M_{14}\right) \exp \left(-\beta_{2} M_{24}\right) \\
& \times \exp \left(-\beta_{3} M_{34}\right) p_{1}^{A_{04}-1} p_{2}^{B_{04}-1}\left(1-p_{1}-p_{2}\right)^{C_{04}-1}
\end{aligned}
$$

where

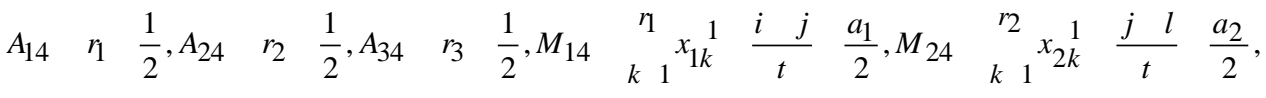

$$
\begin{aligned}
& M_{34} \square \underset{k \square 1}{r_{3}} x_{3 k}^{\square 1} \square \frac{l}{t} \square \frac{a_{3}}{2}, A_{04} \square i \square j \square r_{1} \square a, B_{04} \square j \square l \square r_{2} \square b, C_{04} \square l \square r_{3} \square c,
\end{aligned}
$$

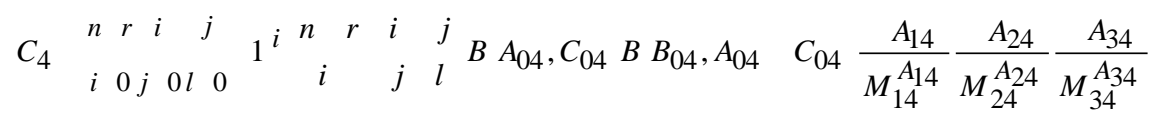

\section{Bayes estimators and posterior risks using the UP, the JP, the Exponential and Inverse Levy prior under SELF, PLF and DLF}

If $\hat{d}$ is a Bayes estimator, then $\mathbb{C} \mathbb{d} \hat{d}[$ is called posterior risk and is defined as:

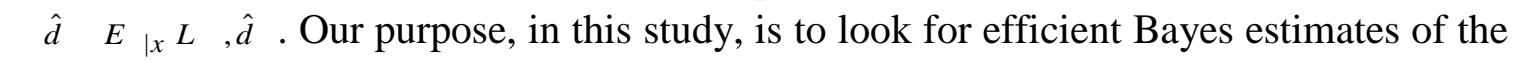
different parameters. For this purpose, three different loss functions, namely SELF, PLF and DLF used to obtain Bayes estimators and their posterior risks. The SELF, defined as

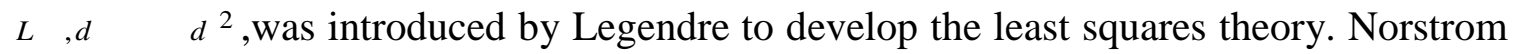
(1996) discussed an asymmetric PLF and also introduced a special case of general class of PLFs, which is defined as $L\left[\square, d \square \frac{\square \square \square d[\text { द }}{d}\right.$. While the DLF is presented by DeGroot (2005) and is defined as $L \square], d \square \square\left[\left.\frac{\square}{d}\right|^{2}\right.$ For a given prior, the Bayes estimator and

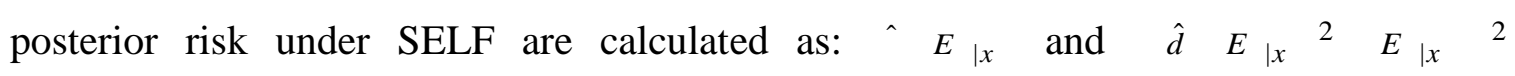
,respectively. Similarly, the Bayes estimators and posterior risks with PLF and DLF are

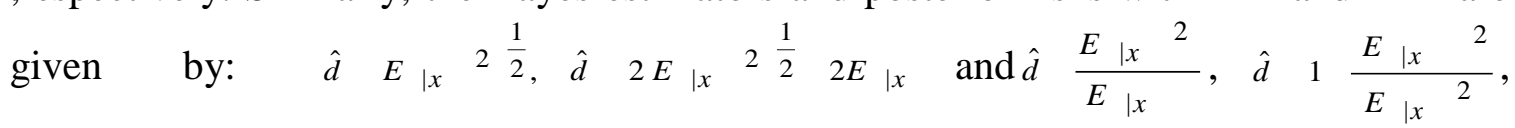
respectively. 
4.1. The Bayes estimators and posterior risks using the UP, the JP and IP under SELF. The Bayes estimators and posterior risks using the UP, the JP and IP for parameters $\beta_{1}, \beta_{2}, \beta_{3}, p_{1}$ and $p_{2}$ under SELF are obtained with their respective marginal posterior distributions are given below:

$$
\begin{aligned}
& \hat{\beta}_{1 v}=C_{v}^{-1} \sum_{i=0}^{n-r} \sum_{j=0}^{i} \sum_{l=0}^{j}(-1)^{i}\left(\begin{array}{c}
n-r \\
i
\end{array}\right)\left(\begin{array}{l}
i \\
j
\end{array}\right)\left(\begin{array}{l}
j \\
l
\end{array}\right) \frac{\Gamma\left(A_{1 v}+1\right)}{M_{1 v}^{A_{1 v}+1}} \frac{\Gamma\left(A_{2 v}\right)}{M_{2 v}^{A_{2 v}}} \\
& \times \frac{\Gamma\left(A_{3 v}\right)}{M_{3 v}^{A_{3 v}}} B\left(A_{0 v}, C_{0 v}\right) B\left(B_{0 v}, A_{0 v}+C_{0 v}\right) \\
& \hat{\beta}_{2 v}=C_{v}^{-1} \sum_{i=0}^{n-r} \sum_{j=0}^{i} \sum_{l=0}^{j}(-1)^{i}\left(\begin{array}{c}
n-r \\
i
\end{array}\right)\left(\begin{array}{l}
i \\
j
\end{array}\right)\left(\begin{array}{l}
j \\
l
\end{array}\right) \frac{\Gamma\left(A_{1 v}\right)}{M_{1 v}^{A_{1 v}}} \frac{\Gamma\left(A_{2 v}+1\right)}{M_{2 v}^{A_{2 v}+1}} \\
& \times \frac{\Gamma\left(A_{3 v}\right)}{M_{3 v}^{A_{3 v}}} B\left(A_{0 v}, C_{0 v}\right) B\left(B_{0 v}, A_{0 v}+C_{0 v}\right) \\
& \hat{\beta}_{3 v}=C_{v}^{-1} \sum_{i=0}^{n-r} \sum_{j=0}^{i} \sum_{l=0}^{j}(-1)^{i}\left(\begin{array}{c}
n-r \\
i
\end{array}\right)\left(\begin{array}{l}
i \\
j
\end{array}\right)\left(\begin{array}{l}
j \\
l
\end{array}\right) \frac{\Gamma\left(A_{1 v}\right)}{M_{1 v}^{A_{1 v}}} \frac{\Gamma\left(A_{2 v}\right)}{M_{2 v}^{A_{2 v}}} \\
& \times \frac{\Gamma\left(A_{3 v}+1\right)}{M_{3 v}^{A_{3 v}+1}} B\left(A_{0 v}, C_{0 v}\right) B\left(B_{0 v}, A_{0 v}+C_{0 v}\right) \\
& \hat{p}_{1 v}=C_{v}^{-1} \sum_{i=0}^{n-r} \sum_{j=0}^{i} \sum_{l=0}^{j}(-1)^{i}\left(\begin{array}{c}
n-r \\
i
\end{array}\right)\left(\begin{array}{l}
i \\
j
\end{array}\right)\left(\begin{array}{l}
j \\
l
\end{array}\right) \frac{\Gamma\left(A_{1 v}\right)}{M_{1 v}^{A_{1 v}}} \frac{\Gamma\left(A_{2 v}\right)}{M_{2 v}^{A_{2 v}}} \frac{\Gamma\left(A_{3 v}\right)}{M_{3 v}^{A_{3 v}}} \\
& \times B\left(B_{0 v}, C_{0 v}\right) B\left(A_{0 v}+1, B_{0 v}+C_{0 v}\right) \\
& \hat{p}_{2 v}=C_{v}^{-1} \sum_{i=0}^{n-r} \sum_{j=0}^{i} \sum_{l=0}^{j}(-1)^{i}\left(\begin{array}{c}
n-r \\
i
\end{array}\right)\left(\begin{array}{l}
i \\
j
\end{array}\right)\left(\begin{array}{l}
j \\
l
\end{array}\right) \frac{\Gamma\left(A_{1 v}\right)}{M_{1 v}^{A_{1 v}}} \frac{\Gamma\left(A_{2 v}\right)}{M_{2 v}^{A_{2 v}}} \frac{\Gamma\left(A_{3 v}\right)}{M_{3 v}^{A_{3 v}}} \\
& \times B\left(A_{0 v}, C_{0 v}\right) B\left(B_{0 v}+1, A_{0 v}+C_{0 v}\right) \\
& \rho\left(\hat{\beta}_{1 v}\right)=C_{v}^{-1} \sum_{i=0}^{n-r} \sum_{j=0}^{i} \sum_{l=0}^{j}(-1)^{i}\left(\begin{array}{c}
n-r \\
i
\end{array}\right)\left(\begin{array}{l}
i \\
j
\end{array}\right)\left(\begin{array}{l}
j \\
l
\end{array}\right) \frac{\Gamma\left(A_{1 v}+2\right)}{M_{1 v}^{A_{1 v}+2}} \frac{\Gamma\left(A_{2 v}\right)}{M_{2 v}^{A_{2 v}}} \\
& \times \frac{\Gamma\left(A_{3 v}\right)}{M_{3 v}^{A_{3 v}}} B\left(A_{0 v}, C_{0 v}\right) B\left(B_{0 v}, A_{0 v}+C_{0 v}\right)-\left(\hat{\beta}_{1 v}\right)^{2} \\
& \rho\left(\hat{\beta}_{2 v}\right)=C_{v}^{-1} \sum_{i=0}^{n-r} \sum_{j=0}^{i} \sum_{l=0}^{j}(-1)^{i}\left(\begin{array}{c}
n-r \\
i
\end{array}\right)\left(\begin{array}{c}
i \\
j
\end{array}\right)\left(\begin{array}{l}
j \\
l
\end{array}\right) \frac{\Gamma\left(A_{1 v}\right)}{M_{1 v}^{A_{1 v}}} \frac{\Gamma\left(A_{2 v}+2\right)}{M_{2 v}^{A_{2 v}+2}} \\
& \times \frac{\Gamma\left(A_{3 v}\right)}{M_{3 v}^{A_{3 v}}} B\left(A_{0 v}, C_{0 v}\right) B\left(B_{0 v}, A_{0 v}+C_{0 v}\right)-\left(\hat{\beta}_{2 v}\right)^{2} \\
& \rho\left(\hat{\beta}_{3 v}\right)=C_{v}^{-1} \sum_{i=0}^{n-r} \sum_{j=0}^{i} \sum_{l=0}^{j}(-1)^{i}\left(\begin{array}{c}
n-r \\
i
\end{array}\right)\left(\begin{array}{c}
i \\
j
\end{array}\right)\left(\begin{array}{l}
j \\
l
\end{array}\right) \frac{\Gamma\left(A_{1 v}\right)}{M_{1 v}^{A_{1 v}}} \frac{\Gamma\left(A_{2 v}\right)}{M_{2 v}^{A_{2 v}}} \\
& \times \frac{\Gamma\left(A_{3 v}+2\right)}{M_{3 v}^{A_{3 v}+2}} B\left(A_{0 v}, C_{0 v}\right) B\left(B_{0 v}, A_{0 v}+C_{0 v}\right)-\left(\hat{\beta}_{3 v}\right)^{2} \\
& \rho\left(\hat{p}_{1 v}\right)=C_{v}^{-1} \sum_{i=0}^{n-r} \sum_{j=0}^{i} \sum_{l=0}^{j}(-1)^{i}\left(\begin{array}{c}
n-r \\
i
\end{array}\right)\left(\begin{array}{c}
i \\
j
\end{array}\right)\left(\begin{array}{l}
j \\
l
\end{array}\right) \frac{\Gamma\left(A_{1 v}\right)}{M_{1 v}^{A_{1 v}}} \frac{\Gamma\left(A_{2 v}\right)}{M_{2 v}^{A_{2 v}}} \\
& \times \frac{\Gamma\left(A_{3 v}\right)}{M_{3 v}^{A_{3 v}}} B\left(B_{0 v}, C_{0 v}\right) B\left(A_{0 v}+2, B_{0 v}+C_{0 v}\right)-\left(\hat{p}_{1 v}\right)^{2}
\end{aligned}
$$




$$
\begin{aligned}
\rho\left(\hat{p}_{2 v}\right)= & C_{v}^{-1} \sum_{i=0}^{n-r} \sum_{j=0}^{i} \sum_{l=0}^{j}(-1)^{i}\left(\begin{array}{c}
n-r \\
i
\end{array}\right)\left(\begin{array}{l}
i \\
j
\end{array}\right)\left(\begin{array}{l}
j \\
l
\end{array}\right) \frac{\Gamma\left(A_{1 v}\right)}{M_{1 v}^{A_{1 v}}} \frac{\Gamma\left(A_{2 v}\right)}{M_{2 v}^{A_{2 v}}} \\
& \times \frac{\Gamma\left(A_{3 v}\right)}{M_{3 v}^{A_{3 v}}} B\left(A_{0 v}, C_{0 v}\right) B\left(B_{0 v}+2, A_{0 v}+C_{0 v}\right)-\left(\hat{p}_{2 v}\right)^{2}
\end{aligned}
$$

where $v=1$ for the UP, $v=2$ for the JP, $v=3$ for the EP and $v=4$ for the ILP.

4.2. The Bayes estimators and posterior risks using the UP, the JP and IP under PLF. Norstrom (1996) discussed an asymmetric PLF and also introduced a special case of general class of PLFs, which is defined as $L \llbracket\rceil, d \square \frac{\square\rceil \square d \llbracket}{d}$. The Bayes estimator and posterior risk are:

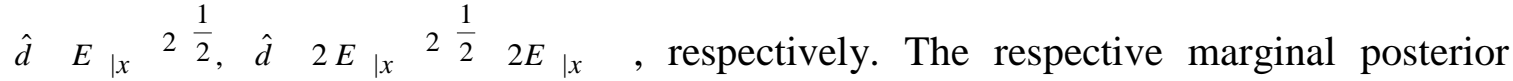
distribution yields the Bayes estimators and posterior risk using the UP, the JP and the IP for parameters $\square_{1}, \square_{2}, \square_{3}, p_{1}$ and $p_{2}$ under PLF as:

$$
\begin{aligned}
& \hat{\beta}_{1 v}=\left\{C_{v}^{-1} \sum_{i=0}^{n-r} \sum_{j=0}^{i} \sum_{l=0}^{j}(-1)^{i}\left(\begin{array}{c}
n-r \\
i
\end{array}\right)\left(\begin{array}{c}
i \\
j
\end{array}\right)\left(\begin{array}{l}
j \\
l
\end{array}\right) \frac{\Gamma\left(A_{1 v}+2\right)}{M_{1 v}^{A_{1 v}+2}}\right. \\
& \left.\frac{\Gamma\left(A_{2 v}\right)}{M_{2 v}^{A_{2 v}}} \frac{\Gamma\left(A_{3 v}\right)}{M_{3 v}^{A_{3 v}}} B\left(A_{0 v}, C_{0 v}\right) B\left(B_{0 v}, A_{0 v}+C_{0 v}\right)\right\}^{\frac{1}{2}} \\
& \hat{\beta}_{2 v}=\left\{C_{v}^{-1} \sum_{i=0}^{n-r} \sum_{j=0}^{i} \sum_{l=0}^{j}(-1)^{i}\left(\begin{array}{c}
n-r \\
i
\end{array}\right)\left(\begin{array}{l}
i \\
j
\end{array}\right)\left(\begin{array}{l}
j \\
l
\end{array}\right) \frac{\Gamma\left(A_{1 v}\right)}{M_{1 v}^{A_{1 v}}}\right. \\
& \left.\frac{\Gamma\left(A_{2 v}+2\right)}{M_{2 v}^{A_{2 v}+2}} \frac{\Gamma\left(A_{3 v}\right)}{M_{3 v}^{A_{3 v}}} B\left(A_{0 v}, C_{0 v}\right) B\left(B_{0 v}, A_{0 v}+C_{0 v}\right)\right\}^{\frac{1}{2}} \\
& \hat{\beta}_{3 v}=\left\{C_{v}^{-1} \sum_{i=0}^{n-r} \sum_{j=0}^{i} \sum_{l=0}^{j}(-1)^{i}\left(\begin{array}{c}
n-r \\
i
\end{array}\right)\left(\begin{array}{l}
i \\
j
\end{array}\right)\left(\begin{array}{l}
j \\
l
\end{array}\right) \frac{\Gamma\left(A_{1 v}\right)}{M_{1 v}^{A_{1 v}}}\right. \\
& \left.\frac{\Gamma\left(A_{2 v}\right)}{M_{2 v}^{A_{2 v}}} \frac{\Gamma\left(A_{3 v}+2\right)}{M_{3 v}^{A_{3 v}+2}} B\left(A_{0 v}, C_{0 v}\right) B\left(B_{0 v}, A_{0 v}+C_{0 v}\right)\right\}^{\frac{1}{2}} \\
& \hat{p}_{1 v}=\left\{C_{v}^{-1} \sum_{i=0}^{n-r} \sum_{j=0}^{i} \sum_{l=0}^{j}(-1)^{i}\left(\begin{array}{c}
n-r \\
i
\end{array}\right)\left(\begin{array}{c}
i \\
j
\end{array}\right)\left(\begin{array}{l}
j \\
l
\end{array}\right) \frac{\Gamma\left(A_{1 v}\right)}{M_{1 v}^{A_{1 v}}} \frac{\Gamma\left(A_{2 v}\right)}{M_{2 v}^{A_{2 v}}}\right. \\
& \left.\frac{\Gamma\left(A_{3 v}\right)}{M_{3 v}^{A_{3 v}}} B\left(B_{0 v}, C_{0 v}\right) B\left(A_{0 v}+2, B_{0 v}+C_{0 v}\right)\right\}^{\frac{1}{2}} \\
& \hat{p}_{2 v}=\left\{C_{v}^{-1} \sum_{i=0}^{n-r} \sum_{j=0}^{i} \sum_{l=0}^{j}(-1)^{i}\left(\begin{array}{c}
n-r \\
i
\end{array}\right)\left(\begin{array}{c}
i \\
j
\end{array}\right)\left(\begin{array}{l}
j \\
l
\end{array}\right) \frac{\Gamma\left(A_{1 v}\right)}{M_{1 v}^{A_{1 v}}} \frac{\Gamma\left(A_{2 v}\right)}{M_{2 v}^{A_{2 v}}}\right. \\
& \left.\frac{\Gamma\left(A_{3 v}\right)}{M_{3 v}^{A_{3 v}}} B\left(A_{0 v}, C_{0 v}\right) B\left(B_{0 v}+2, A_{0 v}+C_{0 v}\right)\right\}^{\frac{1}{2}}
\end{aligned}
$$




$$
\begin{aligned}
& \rho\left(\hat{\beta}_{1 v}\right)=2\left\{C_{v}^{-1} \sum_{i=0}^{n-r} \sum_{j=0}^{i} \sum_{l=0}^{j}(-1)^{i}\left(\begin{array}{c}
n-r \\
i
\end{array}\right)\left(\begin{array}{c}
i \\
j
\end{array}\right)\left(\begin{array}{l}
j \\
l
\end{array}\right) \frac{\Gamma\left(A_{1 v}+2\right)}{M_{1 v}^{A_{1 v}+2}}\right. \\
& \left.\frac{\Gamma\left(A_{2 v}\right)}{M_{2 v}^{A_{2 v}}} \frac{\Gamma\left(A_{3 v}\right)}{M_{3 v}^{A_{3 v}}} B\left(A_{0 v}, C_{0 v}\right) B\left(B_{0 v}, A_{0 v}+C_{0 v}\right)\right\}^{\frac{1}{2}} \\
& -2\left\{C_{v}^{-1} \sum_{i=0}^{n-r} \sum_{j=0}^{i} \sum_{l=0}^{j}(-1)^{i}\left(\begin{array}{c}
n-r \\
i
\end{array}\right)\left(\begin{array}{c}
i \\
j
\end{array}\right)\left(\begin{array}{l}
j \\
l
\end{array}\right) \frac{\Gamma\left(A_{1 v}+1\right)}{M_{1 v}^{A_{1 v}+1}}\right. \\
& \left.\frac{\Gamma\left(A_{2 v}\right)}{M_{2 v}^{A_{2 v}}} \frac{\Gamma\left(A_{3 v}\right)}{M_{3 v}^{A_{3 v}}} B\left(A_{0 v}, C_{0 v}\right) B\left(B_{0 v}, A_{0 v}+C_{0 v}\right)\right\} \\
& \rho\left(\hat{\beta}_{2 v}\right)=2\left\{C_{v}^{-1} \sum_{i=0}^{n-r} \sum_{j=0}^{i} \sum_{l=0}^{j}(-1)^{i}\left(\begin{array}{c}
n-r \\
i
\end{array}\right)\left(\begin{array}{c}
i \\
j
\end{array}\right)\left(\begin{array}{l}
j \\
l
\end{array}\right) \frac{\Gamma\left(A_{1 v}\right)}{M_{1 v}^{A_{1 v}}}\right. \\
& \left.\frac{\Gamma\left(A_{2 v}+2\right)}{M_{2 v}^{A_{2 v}+2}} \frac{\Gamma\left(A_{3 v}\right)}{M_{3 v}^{A_{3 v}}} B\left(A_{0 v}, C_{0 v}\right) B\left(B_{0 v}, A_{0 v}+C_{0 v}\right)\right\}^{\frac{1}{2}} \\
& -2\left\{C_{v}^{-1} \sum_{i=0}^{n-r} \sum_{j=0}^{i} \sum_{l=0}^{j}(-1)^{i}\left(\begin{array}{c}
n-r \\
i
\end{array}\right)\left(\begin{array}{c}
i \\
j
\end{array}\right)\left(\begin{array}{l}
j \\
l
\end{array}\right) \frac{\Gamma\left(A_{1 v}\right)}{M_{1 v}^{A_{1 v}}}\right. \\
& \left.\frac{\Gamma\left(A_{2 v}+1\right)}{M_{2 v}^{A_{2 v}+1}} \frac{\Gamma\left(A_{3 v}\right)}{M_{3 v}^{A_{3 v}}} B\left(A_{0 v}, C_{0 v}\right) B\left(B_{0 v}, A_{0 v}+C_{0 v}\right)\right\} \\
& \rho\left(\hat{\beta}_{3 v}\right)=2\left\{C_{v}^{-1} \sum_{i=0}^{n-r} \sum_{j=0}^{i} \sum_{l=0}^{j}(-1)^{i}\left(\begin{array}{c}
n-r \\
i
\end{array}\right)\left(\begin{array}{l}
i \\
j
\end{array}\right)\left(\begin{array}{l}
j \\
l
\end{array}\right) \frac{\Gamma\left(A_{1 v}\right)}{M_{1 v}^{A_{1 v}}}\right. \\
& \left.\frac{\Gamma\left(A_{2 v}\right)}{M_{2 v}^{A_{2 v}+2}} \frac{\Gamma\left(A_{3 v}+2\right)}{M_{3 v}^{A_{3 v}+2}} B\left(A_{0 v}, C_{0 v}\right) B\left(B_{0 v}, A_{0 v}+C_{0 v}\right)\right\}^{\frac{1}{2}} \\
& -2\left\{C_{v}^{-1} \sum_{i=0}^{n-r} \sum_{j=0}^{i} \sum_{l=0}^{j}(-1)^{i}\left(\begin{array}{c}
n-r \\
i
\end{array}\right)\left(\begin{array}{c}
i \\
j
\end{array}\right)\left(\begin{array}{l}
j \\
l
\end{array}\right) \frac{\Gamma\left(A_{1 v}\right)}{M_{1 v}^{A_{1 v}}}\right. \\
& \left.\frac{\Gamma\left(A_{2 v}\right)}{M_{2 v}^{A_{2 v}}} \frac{\Gamma\left(A_{3 v}+1\right)}{M_{3 v}^{A_{3 v}+1}} B\left(A_{0 v}, C_{0 v}\right) B\left(B_{0 v}, A_{0 v}+C_{0 v}\right)\right\} \\
& \rho\left(\hat{p}_{1 v}\right)=2\left\{C_{v}^{-1} \sum_{i=0}^{n-r} \sum_{j=0}^{i} \sum_{l=0}^{j}(-1)^{i}\left(\begin{array}{c}
n-r \\
i
\end{array}\right)\left(\begin{array}{l}
i \\
j
\end{array}\right)\left(\begin{array}{l}
j \\
l
\end{array}\right) \frac{\Gamma\left(A_{1 v}\right)}{M_{1 v}^{A_{1 v}}}\right. \\
& \left.\frac{\Gamma\left(A_{2 v}\right)}{M_{2 v}^{A_{2 v}+2}} \frac{\Gamma\left(A_{3 v}\right)}{M_{3 v}^{A_{3 v}}} B\left(B_{0 v}, C_{0 v}\right) B\left(A_{0 v}+2, B_{0 v}+C_{0 v}\right)\right\}^{\frac{1}{2}} \\
& -2\left\{C_{v}^{-1} \sum_{i=0}^{n-r} \sum_{j=0}^{i} \sum_{l=0}^{j}(-1)^{i}\left(\begin{array}{c}
n-r \\
i
\end{array}\right)\left(\begin{array}{l}
i \\
j
\end{array}\right)\left(\begin{array}{l}
j \\
l
\end{array}\right) \frac{\Gamma\left(A_{1 v}\right)}{M_{1 v}^{A_{1 v}}}\right. \\
& \left.\frac{\Gamma\left(A_{2 v}\right)}{M_{2 v}^{A_{2 v}}} \frac{\Gamma\left(A_{3 v}\right)}{M_{3 v}^{A_{3 v}}} B\left(B_{0 v}, C_{0 v}\right) B\left(A_{0 v}+1, B_{0 v}+C_{0 v}\right)\right\}
\end{aligned}
$$




$$
\begin{aligned}
& \rho\left(\hat{p}_{2 v}\right)=2\left\{C_{v}^{-1} \sum_{i=0}^{n-r} \sum_{j=0}^{i} \sum_{l=0}^{j}(-1)^{i}\left(\begin{array}{c}
n-r \\
i
\end{array}\right)\left(\begin{array}{l}
i \\
j
\end{array}\right)\left(\begin{array}{l}
j \\
l
\end{array}\right) \frac{\Gamma\left(A_{1 v}\right)}{M_{1 v}^{A_{1 v}}}\right. \\
& \left.\frac{\Gamma\left(A_{2 v}\right)}{M_{2 v}^{A_{2 v}+2}} \frac{\Gamma\left(A_{3 v}\right)}{M_{3 v}^{A_{3 v}}} B\left(A_{0 v}, C_{0 v}\right) B\left(B_{0 v}+2, A_{0 v}+C_{0 v}\right)\right\}^{\frac{1}{2}} \\
& -2\left\{C_{v}^{-1} \sum_{i=0}^{n-r} \sum_{j=0}^{i} \sum_{l=0}^{j}(-1)^{i}\left(\begin{array}{c}
n-r \\
i
\end{array}\right)\left(\begin{array}{l}
i \\
j
\end{array}\right)\left(\begin{array}{l}
j \\
l
\end{array}\right) \frac{\Gamma\left(A_{1 v}\right)}{M_{1 v}^{A_{1 v}}}\right. \\
& \left.\frac{\Gamma\left(A_{2 v}\right)}{M_{2 v}^{A_{2 v}}} \frac{\Gamma\left(A_{3 v}\right)}{M_{3 v}^{A_{3 v}}} B\left(A_{0 v}, C_{0 v}\right) B\left(B_{0 v}+1, A_{0 v}+C_{0 v}\right)\right\}
\end{aligned}
$$

4.3. The Bayes estimators and posterior risks using the UP, the JP and IP under DLF. The Bayes estimators and posterior risks using the UP, the JP and the IP for parameters $\square_{1}, \square_{2}, \square_{3}, p_{1}$ and $p_{2}$ under DLF are:

$$
\begin{aligned}
& \left\{C_{v}^{-1} \sum_{i=0}^{n-r} \sum_{j=0}^{i} \sum_{l=0}^{j}(-1)^{i}\left(\begin{array}{c}
n-r \\
i
\end{array}\right)\left(\begin{array}{l}
i \\
j
\end{array}\right)\left(\begin{array}{l}
j \\
l
\end{array}\right) \frac{\Gamma\left(A_{1 v}+2\right)}{M_{1 v}^{A_{1 v}+2}}\right. \\
& \hat{\beta}_{1 v}=\frac{\left.\frac{\Gamma\left(A_{2 v}\right)}{M_{2 v}^{A_{2 v}}} \frac{\Gamma\left(A_{3 v}\right)}{M_{3 v}^{A_{3 v}}} B\left(A_{0 v}, C_{0 v}\right) B\left(B_{0 v}, A_{0 v}+C_{0 v}\right)\right\}}{\left\{C_{v}^{-1} \sum_{i=0}^{n-r} \sum_{j=0}^{i} \sum_{l=0}^{j}(-1)^{i}\left(\begin{array}{c}
n-r \\
i
\end{array}\right)\left(\begin{array}{c}
i \\
j
\end{array}\right)\left(\begin{array}{c}
j \\
l
\end{array}\right) \frac{\Gamma\left(A_{1 v}+1\right)}{M_{1 v}^{A_{1 v}+1}}\right.} \\
& \left.\frac{\Gamma\left(A_{2 v}\right)}{M_{2 v}^{A_{2 v}}} \frac{\Gamma\left(A_{3 v}\right)}{M_{3 v}^{A_{3 v}}} B\left(A_{0 v}, C_{0 v}\right) B\left(B_{0 v}, A_{0 v}+C_{0 v}\right)\right\} \\
& \left\{C_{v}^{-1} \sum_{i=0}^{n-r} \sum_{j=0}^{i} \sum_{l=0}^{j}(-1)^{i}\left(\begin{array}{c}
n-r \\
i
\end{array}\right)\left(\begin{array}{l}
i \\
j
\end{array}\right)\left(\begin{array}{l}
j \\
l
\end{array}\right) \frac{\Gamma\left(A_{1 v}\right)}{M_{1 v}^{A_{1 v}}}\right. \\
& \hat{\beta}_{2 v}=\frac{\left.\frac{\Gamma\left(A_{2 v}+2\right)}{M_{2 v}^{A_{2 v}+2}} \frac{\Gamma\left(A_{3 v}\right)}{M_{3 v}^{A_{3 v}}} B\left(A_{0 v}, C_{0 v}\right) B\left(B_{0 v}, A_{0 v}+C_{0 v}\right)\right\}}{\left\{C_{v}^{-1} \sum_{i=0}^{n-r} \sum_{j=0}^{i} \sum_{l=0}^{j}(-1)^{i}\left(\begin{array}{c}
n-r \\
i
\end{array}\right)\left(\begin{array}{c}
i \\
j
\end{array}\right)\left(\begin{array}{l}
j \\
l
\end{array}\right) \frac{\Gamma\left(A_{1 v}\right)}{M_{1 v}^{A_{1 v}}}\right.} \\
& \left.\frac{\Gamma\left(A_{2 v}+1\right)}{M_{2 v}^{A_{2 v}+1}} \frac{\Gamma\left(A_{3 v}\right)}{M_{3 v}^{A_{3 v}}} B\left(A_{0 v}, C_{0 v}\right) B\left(B_{0 v}, A_{0 v}+C_{0 v}\right)\right\}
\end{aligned}
$$




$$
\begin{aligned}
& \left\{C_{v}^{-1} \sum_{i=0}^{n-r} \sum_{j=0}^{i} \sum_{l=0}^{j}(-1)^{i}\left(\begin{array}{c}
n-r \\
i
\end{array}\right)\left(\begin{array}{l}
i \\
j
\end{array}\right)\left(\begin{array}{l}
j \\
l
\end{array}\right) \frac{\Gamma\left(A_{1 v}\right)}{M_{1 v}^{A_{1 v}}}\right. \\
& \hat{\beta}_{3 v}=\frac{\left.\frac{\Gamma\left(A_{2 v}\right)}{M_{2 v}^{A_{2 v}}} \frac{\Gamma\left(A_{3 v}+2\right)}{M_{3 v}^{A_{3 v}+2}} B\left(A_{0 v}, C_{0 v}\right) B\left(B_{0 v}, A_{0 v}+C_{0 v}\right)\right\}}{\left\{C_{v}^{-1} \sum_{i=0}^{n-r} \sum_{j=0}^{i} \sum_{l=0}^{j}(-1)^{i}\left(\begin{array}{c}
n-r \\
i
\end{array}\right)\left(\begin{array}{c}
i \\
j
\end{array}\right)\left(\begin{array}{l}
j \\
l
\end{array}\right) \frac{\Gamma\left(A_{1 v}\right)}{M_{1 v}^{A_{1 v}}}\right.} \\
& \left.\frac{\Gamma\left(A_{2 v}+1\right)}{M_{2 v}^{A_{2 v}+1}} \frac{\Gamma\left(A_{3 v}+1\right)}{M_{3 v}^{A_{3 v}+1}} B\left(A_{0 v}, C_{0 v}\right) B\left(B_{0 v}, A_{0 v}+C_{0 v}\right)\right\} \\
& \left\{C_{v}^{-1} \sum_{i=0}^{n-r} \sum_{j=0}^{i} \sum_{l=0}^{j}(-1)^{i}\left(\begin{array}{c}
n-r \\
i
\end{array}\right)\left(\begin{array}{l}
i \\
j
\end{array}\right)\left(\begin{array}{l}
j \\
l
\end{array}\right) \frac{\Gamma\left(A_{1 v}\right)}{M_{1 v}^{A_{1 v}}}\right. \\
& \hat{p}_{1 v}=\frac{\left.\frac{\Gamma\left(A_{2 v}\right)}{M_{2 v}^{A_{2 v}}} \frac{\Gamma\left(A_{3 v}\right)}{B_{3 v}^{A_{3 v}}} B\left(B_{0 v}, C_{0 v}\right) B\left(A_{0 v}+2, B_{0 v}+C_{0 v}\right)\right\}}{\left\{C_{v}^{-1} \sum_{i=0}^{n-r} \sum_{j=0}^{i} \sum_{l=0}^{j}(-1)^{i}\left(\begin{array}{c}
n-r \\
i
\end{array}\right)\left(\begin{array}{l}
i \\
j
\end{array}\right)\left(\begin{array}{l}
j \\
l
\end{array}\right) \frac{\Gamma\left(A_{1 v}\right)}{M_{1 v}^{A_{1 v}}}\right.} \\
& \left.\frac{\Gamma\left(A_{2 v}\right)}{M_{2 v}^{A_{2 v}}} \frac{\Gamma\left(A_{3 v}\right)}{M_{3 v}^{A_{3 v}}} B\left(B_{0 v}, C_{0 v}\right) B\left(A_{0 v}+1, B_{0 v}+C_{0 v}\right)\right\} \\
& \left\{C_{v}^{-1} \sum_{i=0}^{n-r} \sum_{j=0}^{i} \sum_{l=0}^{j}(-1)^{i}\left(\begin{array}{c}
n-r \\
i
\end{array}\right)\left(\begin{array}{l}
i \\
j
\end{array}\right)\left(\begin{array}{l}
j \\
l
\end{array}\right) \frac{\Gamma\left(A_{1 v}\right)}{M_{1 v}^{A_{1 v}}}\right. \\
& \hat{p}_{2 v}=\frac{\left.\frac{\Gamma\left(A_{2 v}\right)}{M_{2 v}^{A_{2 v}}} \frac{\Gamma\left(A_{3 v}\right)}{B_{3 v}^{A_{3 v}}} B\left(A_{0 v}, C_{0 v}\right) B\left(B_{0 v}+2, A_{0 v}+C_{0 v}\right)\right\}}{\left\{C_{v}^{-1} \sum_{i=0}^{n-r} \sum_{j=0}^{i} \sum_{l=0}^{j}(-1)^{i}\left(\begin{array}{c}
n-r \\
i
\end{array}\right)\left(\begin{array}{c}
i \\
j
\end{array}\right)\left(\begin{array}{c}
j \\
l
\end{array}\right) \frac{\Gamma\left(A_{1 v}\right)}{M_{1 v}^{A_{1 v}}}\right.} \\
& \left.\frac{\Gamma\left(A_{2 v}\right)}{M_{2 v}^{A_{2 v}}} \frac{\Gamma\left(A_{3 v}\right)}{B_{3 v}^{A_{3 v}}} B\left(A_{0 v}, C_{0 v}\right) B\left(B_{0 v}+1, A_{0 v}+C_{0 v}\right)\right\}
\end{aligned}
$$




$$
\begin{aligned}
& \left\{C_{v}^{-1} \sum_{i=0}^{n-r} \sum_{j=0}^{i} \sum_{l=0}^{j}(-1)^{i}\left(\begin{array}{c}
n-r \\
i
\end{array}\right)\left(\begin{array}{c}
i \\
j
\end{array}\right)\left(\begin{array}{l}
j \\
l
\end{array}\right) \frac{\Gamma\left(A_{1 v}+1\right)}{M_{1 v}^{A_{1 v}+1}}\right. \\
& \rho\left(\hat{\beta}_{1}\right)=1-\frac{\left.\frac{\Gamma\left(A_{2 v}\right)}{M_{2 v}^{A_{2 v}}} \frac{\Gamma\left(A_{3 v}\right)}{M_{3 v}^{A_{3 v}}} B\left(A_{0 v}, C_{0 v}\right) B\left(B_{0 v}, A_{0 v}+C_{0 v}\right)\right\}^{2}}{\left\{C_{v}^{-1} \sum_{i=0}^{n-r} \sum_{j=0}^{i} \sum_{l=0}^{j}(-1)^{i}\left(\begin{array}{c}
n-r \\
i
\end{array}\right)\left(\begin{array}{c}
i \\
j
\end{array}\right)\left(\begin{array}{c}
j \\
l
\end{array}\right) \frac{\Gamma\left(A_{1 v}+2\right)}{M_{1 v}^{A_{1 v}+2}}\right.} \\
& \left.\frac{\Gamma\left(A_{2 v}\right)}{M_{2 v}^{A_{2 v}}} \frac{\Gamma\left(A_{3 v}\right)}{M_{3 v}^{A_{3 v}}} B\left(A_{0 v}, C_{0 v}\right) B\left(B_{0 v}, A_{0 v}+C_{0 v}\right)\right\} \\
& \left\{C_{v}^{-1} \sum_{i=0}^{n-r} \sum_{j=0}^{i} \sum_{l=0}^{j}(-1)^{i}\left(\begin{array}{c}
n-r \\
i
\end{array}\right)\left(\begin{array}{c}
i \\
j
\end{array}\right)\left(\begin{array}{l}
j \\
l
\end{array}\right) \frac{\Gamma\left(A_{1 v}\right)}{M_{1 v}^{A_{1 v}}}\right. \\
& \rho\left(\hat{\beta}_{2}\right)=1-\frac{\left.\frac{\Gamma\left(A_{2 v}+1\right)}{M_{2 v}^{A_{2 v}+1}} \frac{\Gamma\left(A_{3 v}\right)}{M_{3 v}^{A_{3 v}}} B\left(A_{0 v}, C_{0 v}\right) B\left(B_{0 v}, A_{0 v}+C_{0 v}\right)\right\}^{2}}{\left\{C_{v}^{-1} \sum_{i=0}^{n-r} \sum_{j=0}^{i} \sum_{l=0}^{j}(-1)^{i}\left(\begin{array}{c}
n-r \\
i
\end{array}\right)\left(\begin{array}{c}
i \\
j
\end{array}\right)\left(\begin{array}{c}
j \\
l
\end{array}\right) \frac{\Gamma\left(A_{1 v}\right)}{M_{1 v}^{A_{1 v}}}\right.} \\
& \left.\frac{\Gamma\left(A_{2 v}+2\right)}{M_{2 v}^{A_{2 v}+2}} \frac{\Gamma\left(A_{3 v}\right)}{M_{3 v}^{A_{3 v}}} B\left(A_{0 v}, C_{0 v}\right) B\left(B_{0 v}, A_{0 v}+C_{0 v}\right)\right\} \\
& \left\{C_{v}^{-1} \sum_{i=0}^{n-r} \sum_{j=0}^{i} \sum_{l=0}^{j}(-1)^{i}\left(\begin{array}{c}
n-r \\
i
\end{array}\right)\left(\begin{array}{c}
i \\
j
\end{array}\right)\left(\begin{array}{l}
j \\
l
\end{array}\right) \frac{\Gamma\left(A_{1 v}\right)}{M_{1 v}^{A_{1 v}}}\right. \\
& \rho\left(\hat{\beta}_{3}\right)=1-\frac{\left.\frac{\Gamma\left(A_{2 v}\right)}{M_{2 v}^{A_{2 v}}} \frac{\Gamma\left(A_{3 v}+1\right)}{M_{3 v}^{A_{3 v}+1}} B\left(A_{0 v}, C_{0 v}\right) B\left(B_{0 v}, A_{0 v}+C_{0 v}\right)\right\}^{2}}{\left\{C_{v}^{-1} \sum_{i=0}^{n-r} \sum_{j=0}^{i} \sum_{l=0}^{j}(-1)^{i}\left(\begin{array}{c}
n-r \\
i
\end{array}\right)\left(\begin{array}{c}
i \\
j
\end{array}\right)\left(\begin{array}{c}
j \\
l
\end{array}\right) \frac{\Gamma\left(A_{1 v}\right)}{M_{1 v}^{A_{1 v}}}\right.} \\
& \left.\frac{\Gamma\left(A_{2 v}\right)}{M_{2 v}^{A_{2 v}}} \frac{\Gamma\left(A_{3 v}+2\right)}{M_{3 v}^{A_{3 v}+2}} B\left(A_{0 v}, C_{0 v}\right) B\left(B_{0 v}, A_{0 v}+C_{0 v}\right)\right\} \\
& \left\{C_{v}^{-1} \sum_{i=0}^{n-r} \sum_{j=0}^{i} \sum_{l=0}^{j}(-1)^{i}\left(\begin{array}{c}
n-r \\
i
\end{array}\right)\left(\begin{array}{l}
i \\
j
\end{array}\right)\left(\begin{array}{l}
j \\
l
\end{array}\right) \frac{\Gamma\left(A_{1 v}\right)}{M_{1 v}^{A_{1 v}}}\right. \\
& \rho\left(\hat{p}_{1}\right)=1-\frac{\left.\frac{\Gamma\left(A_{2 v}\right)}{M_{2 v}^{A_{2 v}}} \frac{\Gamma\left(A_{3 v}\right)}{M_{3 v}^{A_{3 v}}} B\left(B_{0 v}, C_{0 v}\right) B\left(A_{0 v}+1, B_{0 v}+C_{0 v}\right)\right\}^{2}}{\left\{C_{v}^{-1} \sum_{i=0}^{n-r} \sum_{j=0}^{i} \sum_{l=0}^{j}(-1)^{i}\left(\begin{array}{c}
n-r \\
i
\end{array}\right)\left(\begin{array}{c}
i \\
j
\end{array}\right)\left(\begin{array}{c}
j \\
l
\end{array}\right) \frac{\Gamma\left(A_{1 v}\right)}{M_{1 v}^{A_{1 v}}}\right.} \\
& \left.\frac{\Gamma\left(A_{2 v}\right)}{M_{2 v}^{A_{2 v}}} \frac{\Gamma\left(A_{3 v}\right)}{M_{3 v}^{A_{3 v}}} B\left(B_{0 v}, C_{0 v}\right) B\left(A_{0 v}+2, B_{0 v}+C_{0 v}\right)\right\}
\end{aligned}
$$




$$
\begin{aligned}
& \left\{C_{v}^{-1} \sum_{i=0}^{n-r} \sum_{j=0}^{i} \sum_{l=0}^{j}(-1)^{i}\left(\begin{array}{c}
n-r \\
i
\end{array}\right)\left(\begin{array}{c}
i \\
j
\end{array}\right)\left(\begin{array}{l}
j \\
l
\end{array}\right) \frac{\Gamma\left(A_{1 v}\right)}{M_{1 v}^{A_{1 v}}}\right. \\
& \rho\left(\hat{p}_{2}\right)=1-\frac{\left.\frac{\Gamma\left(A_{2 v}\right)}{M_{2 v}^{A_{2 v}}} \frac{\Gamma\left(A_{3 v}\right)}{M_{3 v}^{A_{3 v}}} B\left(A_{0 v}, C_{0 v}\right) B\left(B_{0 v}+1, A_{0 v}+C_{0 v}\right)\right\}^{2}}{\left\{C_{v}^{-1} \sum_{i=0}^{n-r} \sum_{j=0}^{i} \sum_{l=0}^{j}(-1)^{i}\left(\begin{array}{c}
n-r \\
i
\end{array}\right)\left(\begin{array}{c}
i \\
j
\end{array}\right)\left(\begin{array}{c}
j \\
l
\end{array}\right) \frac{\Gamma\left(A_{1 v}\right)}{M_{1 v}^{A_{1 v}}}\right.} \\
& \left.\frac{\Gamma\left(A_{2 v}\right)}{M_{2 v}^{A_{2 v}}} \frac{\Gamma\left(A_{3 v}\right)}{M_{3 v}^{A_{3 v}}} B\left(A_{0 v}, C_{0 v}\right) B\left(B_{0 v}+2, A_{0 v}+C_{0 v}\right)\right\}
\end{aligned}
$$

\section{Elicitation of Hyper-parameters}

Elicitation is the main task for subjective Bayesian. The complete procedure for quantifying the prior information in the form of prior distribution is precisely known as the elicitation. Aslam (2003) presented different methods of elicitation based on prior predictive distribution for the elicitation of the hyper-parameters. In this study, we use the method of elicitation using prior predictive distribution based on the predictive probabilities. In this approach, confidence levels of the prior predictive are gained for the particular intervals of the random variables. The set of hyper parameters, for which the difference between the elicited probabilities and the expert predictive probabilities is minimum, is considered.

5.1. Elicitation of hyper-parameters using the Exponential Prior. For eliciting the hyper-parameters, prior predictive distribution (PPD) is used. The PPD for a random variable $\mathrm{X}$ is:

$$
\begin{aligned}
& p(x)=\int_{\phi} p(x \mid \phi) \pi_{3}(\phi) d \phi \\
& p(x)=\frac{1}{x^{2}(a+b+c)}\left[\frac{a k_{1}}{\left(k_{1}+x^{-1}\right)^{2}}+\frac{b k_{2}}{\left(k_{2}+x^{-1}\right)^{2}}+\frac{c k_{3}}{\left(k_{3}+x^{-1}\right)^{2}}\right]
\end{aligned}
$$

We choose the prior predictive probabilities, satisfying the laws of probability, to elicit the hyper-parameters of the prior density. Using the prior predictive distribution, we consider the six intervals $(0,1),(1,2),(2,3),(3,4),(4,5)$ and $(5,6)$ with probabilities 0.73 , $0.11,0.05,0.02,0.02$, and 0.01 respectively, given an expert opinion. The following nine equations are derived from the above information:

$$
\begin{aligned}
& \frac{1}{x^{2}(a+b+c)} \int_{0}^{1}\left[\frac{a k_{1}}{\left(k_{1}+x^{-1}\right)^{2}}+\frac{b k_{2}}{\left(k_{2}+x^{-1}\right)^{2}}+\frac{c k_{3}}{\left(k_{3}+x^{-1}\right)^{2}}\right] d x=0.73 \\
& \frac{1}{x^{2}(a+b+c)} \int_{1}^{2}\left[\frac{a k_{1}}{\left(k_{1}+x^{-1}\right)^{2}}+\frac{b k_{2}}{\left(k_{2}+x^{-1}\right)^{2}}+\frac{c k_{3}}{\left(k_{3}+x^{-1}\right)^{2}}\right] d x=0.11 \\
& \frac{1}{x^{2}(a+b+c)} \int_{2}^{3}\left[\frac{a k_{1}}{\left(k_{1}+x^{-1}\right)^{2}}+\frac{b k_{2}}{\left(k_{2}+x^{-1}\right)^{2}}+\frac{c k_{3}}{\left(k_{3}+x^{-1}\right)^{2}}\right] d x=0.05 \\
& \frac{1}{x^{2}(a+b+c)} \int_{3}^{4}\left[\frac{a k_{1}}{\left(k_{1}+x^{-1}\right)^{2}}+\frac{b k_{2}}{\left(k_{2}+x^{-1}\right)^{2}}+\frac{c k_{3}}{\left(k_{3}+x^{-1}\right)^{2}}\right] d x=0.02
\end{aligned}
$$




$$
\begin{aligned}
& \frac{1}{x^{2}(a+b+c)} \int_{4}^{5}\left[\frac{a k_{1}}{\left(k_{1}+x^{-1}\right)^{2}}+\frac{b k_{2}}{\left(k_{2}+x^{-1}\right)^{2}}+\frac{c k_{3}}{\left(k_{3}+x^{-1}\right)^{2}}\right] d x=0.02 \\
& \frac{1}{x^{2}(a+b+c)} \int_{5}^{6}\left[\frac{a k_{1}}{\left(k_{1}+x^{-1}\right)^{2}}+\frac{b k_{2}}{\left(k_{2}+x^{-1}\right)^{2}}+\frac{c k_{3}}{\left(k_{3}+x^{-1}\right)^{2}}\right] d x=0.01
\end{aligned}
$$

For eliciting the hyper parameters $k_{1}, k_{2}, k_{3}, a, b$ and $c$ and the equations are simultaneously solved through the computer program developed in SAS package using the 'PROC SYSLIN' command, the values of the hyper parameters are found to be 2.0003,3.0030,4.0016,2.0103,1.7607and 1.50 respectively.

5.2. Elicitation of hyper parameters using the Inverse Levy Prior. The PPD using Inverse Levy prior for a random variable $\mathrm{X}$ is given by:

$$
\begin{aligned}
& p(x)=\int_{\phi} p\langle x \mid \phi\rangle \pi_{4}(\phi) d \phi \\
& p(x)=\frac{1}{2 \sqrt{2}(a+b+c) x^{2}}\left[\frac{a \sqrt{a_{1}}}{\left(\frac{a_{1}}{2}+x^{-1}\right)^{3 / 2}}+\frac{b \sqrt{a_{2}}}{\left(\frac{a_{2}}{2}+x^{-1}\right)^{3 / 2}}+\frac{c \sqrt{a_{3}}}{\left(\frac{a_{3}}{2}+x^{-1}\right)^{3 / 2}}\right]
\end{aligned}
$$

Using same canon defined as above for the exponential prior, the values of the hyperparameters $a_{1}, a_{2}, a_{3}, a, b$ and $c$ are 1.9520, 2.5321, 3.7735, 0.2763, 0.1167 and 1.0.

6. Limiting Expressions. Letting $t \rightarrow \infty$, all the observations that are assimilated in our analysis are uncensored and therefore $r$ tends $n, r_{1}$ tends to the unknown $n_{1}, r_{2}$ tends to the unknown $n_{2}$ and $r_{3}$ tends to the unknown $n_{3}$. As a result, the amount of information carried in the sample expands, which results in the depletion of the variances of the estimates. The limiting (complete sample) expressions for Bayes estimators and posterior risks using the UP, the JP, the EP and the ILP under SELF, PLF and DLF are given in the Tables 1-6.

Table 1: Limiting Expressions for the Bayes Estimators as $t \rightarrow \infty$ assuming the UP, the JP and the IP under SELF

\begin{tabular}{|c|c|c|c|c|}
\hline & \multicolumn{4}{|c|}{ Bayes Estimators } \\
\hline Parameters & UP & JP & Exponential prior & Inverse Levy Prior \\
\hline$\beta_{1}$ & $\frac{n_{1}+1}{\sum_{k=1}^{n_{1}} x_{1 k}^{-1}}$ & $\frac{n_{1}}{\sum_{k=1}^{n_{1}} x_{1 k}^{-1}}$ & $\frac{n_{1}+1}{\sum_{k=1}^{n_{1}} x_{1 k}^{-1}+k_{1}}$ & $\frac{n_{1}+\frac{1}{2}}{\left(\sum_{k=1}^{n_{1}} x_{1 k}^{-1}+\frac{a_{1}}{2}\right)}$ \\
\hline$\beta_{2}$ & $\frac{n_{2}+1}{\sum_{k=1}^{n_{2}} x_{2 k}^{-1}}$ & $\frac{n_{2}}{\sum_{k=1}^{n_{2}} x_{2 k}^{-1}}$ & $\frac{n_{2}+1}{\sum_{k=1}^{n_{2}} x_{2 k}^{-1}+k_{2}}$ & $\frac{n_{2}+\frac{1}{2}}{\left(\sum_{k=1}^{n_{2}} x_{2 k}^{-1}+\frac{a_{2}}{2}\right)}$ \\
\hline$\beta_{3}$ & $\frac{n_{3}+1}{\sum_{k=1}^{n_{3}} x_{3 k}^{-1}}$ & $\frac{n_{3}}{\sum_{k=1}^{n} x_{3 k}^{-1}}$ & $\frac{n_{3}+1}{\sum_{k=1}^{n_{3}} x_{3 k}^{-2}+k_{3}}$ & $\frac{n_{3}+\frac{1}{2}}{\left(\sum_{k=1}^{n_{3}} x_{3 k}^{-1}+\frac{a_{3}}{2}\right)}$ \\
\hline$p_{1}$ & $\frac{n_{1} \square 1}{n \square 3}$ & $\frac{n_{1} \square 1}{n \square 3}$ & $\frac{n_{1}+a}{n+a+b+c}$ & $\frac{n_{1}+a}{n+a+b+c}$ \\
\hline$p_{2}$ & $\frac{n_{2} \square 1}{n \square 3}$ & $\frac{n_{2} \square 1}{n \square 3}$ & $\frac{n_{2}+b}{n+a+b+c}$ & $\frac{n_{2}+b}{n+a+b+c}$ \\
\hline
\end{tabular}


Table 2: Limiting Expressions for the Bayes Estimators as $t \rightarrow \infty$ using the UP and the JP under PLF

\begin{tabular}{|c|c|c|}
\hline & \multicolumn{2}{|c|}{ Bayes Estimators } \\
\hline Parameters & UP & JP \\
\hline$\beta_{1}$ & $\frac{\left[\left(n_{1}+1\right)\left(n_{1}+2\right)\right]^{\frac{1}{2}}}{\sum_{k=1}^{n_{1}} x_{1 k}^{-1}}$ & $\frac{\left[n_{1}\left(n_{1}+1\right)\right]^{\frac{1}{2}}}{\sum_{k=1}^{n_{1}} x_{1 k}^{-1}}$ \\
\hline$\beta_{2}$ & $\frac{\left[\left(n_{2}+1\right)\left(n_{2}+2\right)\right]^{\frac{1}{2}}}{\sum_{k=1}^{n_{2}} x_{2 k}^{-1}}$ & $\frac{\left[n_{2}\left(n_{2}+2\right)\right]^{\frac{1}{2}}}{\sum_{k=1}^{n_{2}} x_{2 k}^{-1}}$ \\
\hline$\beta_{3}$ & $\frac{\left[\left(n_{3}+1\right)\left(n_{3}+2\right)\right]^{\frac{1}{2}}}{\sum_{k=1}^{n_{3}} x_{3 k}^{-1}}$ & $\frac{\left[n_{3}\left(n_{3}+2\right)\right]^{\frac{1}{2}}}{\sum_{k=1}^{n_{3}} x_{3 k}^{-1}}$ \\
\hline$p_{1}$ & {$\left[\frac{\left(n_{1}+1\right)\left(n_{1}+2\right)}{(n+3)(n+4)}\right]^{\frac{1}{2}}$} & {$\left[\frac{\left(n_{1}+1\right)\left(n_{1}+2\right)}{(n+3)(n+4)}\right]^{\frac{1}{2}}$} \\
\hline$p_{2}$ & {$\left[\frac{\left(n_{2}+1\right)\left(n_{2}+2\right)}{(n+3)(n+4)}\right]^{\frac{1}{2}}$} & {$\left[\frac{\left(n_{2}+1\right)\left(n_{2}+2\right)}{(n+3)(n+4)}\right]^{\frac{1}{2}}$} \\
\hline
\end{tabular}

Table 3: Limiting Expressions for the Bayes Estimators as $t \rightarrow \infty$ using the EP and the ILP under PLF

\begin{tabular}{|c|c|c|}
\hline & \multicolumn{2}{|c|}{ Bayes Estimators } \\
\hline Parameters & Exponential Prior & Inverse Levy Prior \\
\hline$\beta_{1}$ & $\frac{\left[\left(n_{1}+1\right)\left(n_{1}+2\right)\right]^{\frac{1}{2}}}{\sum_{k=1}^{n_{1}} x_{1 k}^{-1}+k_{1}}$ & $\frac{\left[\left(n_{1}\right)\left(n_{1}+1\right)\right]^{\frac{1}{2}}}{\sum_{k=1}^{n_{1}} x_{1 k}^{-1}+\frac{a_{1}}{2}}$ \\
\hline$\beta_{2}$ & $\frac{\left[\left(n_{2}+1\right)\left(n_{2}+2\right)\right]^{\frac{1}{2}}}{\sum_{k=1}^{n_{2}} x_{2 k}^{-1}+k_{2}}$ & $\frac{\left[\left(n_{2}\right)\left(n_{2}+1\right)\right]^{\frac{1}{2}}}{\sum_{k=1}^{n_{2}} x_{2 k}^{-1}+\frac{a_{2}}{2}}$ \\
\hline$\beta_{3}$ & $\frac{\left[\left(n_{3}+1\right)\left(n_{3}+2\right)\right]^{\frac{1}{2}}}{\sum_{k=1}^{n} x_{3 k}^{-1}+k_{3}}$ & $\frac{\left[\left(n_{3}\right)\left(n_{3}+1\right)\right]^{\frac{1}{2}}}{\sum_{k=1}^{n} x_{3 k}^{-1}+\frac{a_{3}}{2}}$ \\
\hline$p_{1}$ & {$\left[\frac{\left(n_{1}+a\right)\left(n_{1}+a+1\right)}{(n+a+b+c)(n+a+b+c+1)}\right]^{\frac{1}{2}}$} & {$\left[\frac{\left(n_{1}+a\right)\left(n_{1}+a+1\right)}{(n+a+b+c)(n+a+b+c+1)}\right]^{\frac{1}{2}}$} \\
\hline$p_{2}$ & {$\left[\frac{\left(n_{2}+b\right)\left(n_{2}+b+1\right)}{(n+a+b+c)(n+a+b+c+1)}\right]^{\frac{1}{2}}$} & {$\left[\frac{\left(n_{2}+b\right)\left(n_{2}+b+1\right)}{(n+a+b+c)(n+a+b+c+1)}\right]^{\frac{1}{2}}$} \\
\hline
\end{tabular}

Table 4: Limiting Expressions for the Bayes Estimators as $t \rightarrow \infty$ using the UP and the JP, EP and ILP under DLF

\begin{tabular}{|c|c|c|c|c|}
\hline & \multicolumn{4}{|c|}{ Bayes Estimators } \\
\hline Parameters & UP & JP & Exponential Prior & Inverse Levy Prior \\
\hline$\beta_{1}$ & $\frac{n_{1}+2}{\sum_{k=1}^{n_{1}} x_{1 k}^{-1}}$ & $\frac{n_{1}+1}{\sum_{k=1}^{n_{1}} x_{1 k}^{-1}}$ & $\frac{n_{1}+2}{\sum_{k=1}^{n_{1}} x_{1 k}^{-1}+k_{1}}$ & $\frac{n_{1}+\frac{1}{2}}{\left(\sum_{k=1}^{n_{1}} x_{1 k}^{-1}+\frac{a_{1}}{2}\right)}$ \\
\hline$\beta_{2}$ & $\frac{n_{2}+2}{\sum_{k=1}^{n_{2}} x_{2 k}^{-1}}$ & $\frac{n_{2}+1}{\sum_{k=1}^{n_{2}} x_{2 k}^{-1}}$ & $\frac{n_{2}+2}{\sum_{k=1}^{n_{2}} x_{2 k}^{-1}+k_{2}}$ & $\frac{n_{2}+\frac{1}{2}}{\left(\sum_{k=1}^{n_{2}} x_{2 k}^{-1}+\frac{a_{2}}{2}\right)}$ \\
\hline$\beta_{3}$ & $\frac{n_{3}+2}{\sum_{k=1}^{n_{3}} x_{3 k}^{-1}}$ & $\frac{n_{3}+1}{\sum_{k=1}^{n_{3}} x_{3 k}^{-1}}$ & $\frac{n_{3}+2}{\sum_{k=1}^{n_{3}} x_{3 k}^{-1}+k_{3}}$ & $\frac{n_{3}+12}{\left(\sum_{k=1}^{n_{3}} x_{3 k}^{-1}+\frac{a_{3}}{2}\right)}$ \\
\hline$p_{1}$ & $\frac{n_{1} \square 2}{n \square 4}$ & $\frac{n_{1} \square 2}{n \square 4}$ & $\frac{n_{1}+a+1}{n+a+b+c}$ & $\frac{n_{1}+a+1}{n+a+b+c}$ \\
\hline$p_{2}$ & $\frac{n_{2} \square 2}{n \square 4}$ & $\frac{n_{2} \square 2}{n \square 4}$ & $\frac{n_{2} \square b \square 1}{n \square a \square b \square c}$ & $\frac{n_{2} \square b \square 1}{n \square a \square b \square c}$ \\
\hline
\end{tabular}


Table 5: Limiting Expressions for the Posterior risks as $t \rightarrow \infty$ using the UP and the JP, EP and ILP under SELF

\begin{tabular}{|c|c|c|c|c|}
\hline & \multicolumn{4}{|c|}{ Posterior Risks } \\
\hline Parameters & UP & JP & Exponential Prior & Inverse Levy Prior \\
\hline$\beta_{1}$ & $\frac{n_{1}+1}{\left(\sum_{k=1}^{n_{1}} x_{1 k}^{-1}\right)^{2}}$ & $\frac{n_{1}}{\left(\sum_{k=1}^{n_{1}} x_{1 k}^{-1}\right)^{2}}$ & $\frac{n_{1}+1}{\left(\sum_{k=1}^{n_{1}} x_{1 k}^{-1}+k_{1}\right)^{2}}$ & $\frac{n_{1}+\frac{1}{2}}{\left(\sum_{k=1}^{n_{1}} x_{1 k}^{-1}+\frac{a_{1}}{2}\right)^{2}}$ \\
\hline$\beta_{2}$ & $\frac{n_{2}+1}{\left(\sum_{k=1}^{n_{2}} x_{2 k}^{-1}\right)^{2}}$ & $\frac{n_{2}}{\left(\sum_{k=1}^{n_{2}} x_{2 k}^{-1}\right)^{2}}$ & $\frac{n_{2}+1}{\left(\sum_{k=1}^{n_{2}} x_{2 k}^{-1}+k_{2}\right)^{2}}$ & $\frac{n_{2}+\frac{1}{2}}{\left(\sum_{k=1}^{n_{2}} x_{2 k}^{-1}+\frac{a_{2}}{2}\right)^{2}}$ \\
\hline$\beta_{3}$ & $\frac{n_{3}+1}{\left(\sum_{k=1}^{n_{3}} x_{3 k}^{-1}\right)^{2}}$ & $\frac{n_{3}}{\left(\sum_{k=1}^{n_{3}} x_{3 k}^{-1}\right)^{2}}$ & $\frac{n_{3}+1}{\left(\sum_{k=1}^{n} x_{3 k}^{-1}+k_{3}\right)^{2}}$ & $\frac{n_{3}+\frac{1}{2}}{\left(\sum_{k=1}^{n_{3}} x_{3 k}^{-1}+\frac{a_{3}}{2}\right)^{2}}$ \\
\hline$p_{1}$ & $\frac{\left(n_{1}+1\right)\left(n_{2}+n_{3}+2\right)}{(n+3)^{2}(n+4)}$ & $\frac{\left(n_{1}+1\right)\left(n_{2}+n_{3}+2\right)}{(n+3)^{2}(n+4)}$ & $\frac{\left(n_{1}+1\right)\left(n_{2}+n_{3}+b+c\right)}{(n+a+b+c)^{2}(n+a+b+c+1)}$ & $\frac{\left(n_{1}+1\right)\left(n_{2}+n_{3}+b+c\right)}{(n+a+b+c)^{2}(n+a+b+c+1)}$ \\
\hline$p_{2}$ & $\frac{\left(n_{2}+1\right)\left(n_{2}+n_{3}+2\right)}{(n+3)^{2}(n+4)}$ & $\frac{\left(n_{2}+1\right)\left(n_{2}+n_{3}+2\right)}{(n+3)^{2}(n+4)}$ & $\frac{\left(n_{2}+1\right)\left(n_{1}+n_{3}+b+c\right)}{(n+a+b+c)^{2}(n+a+b+c+1)}$ & $\frac{\left(n_{2}+1\right)\left(n_{1}+n_{3}+b+c\right)}{(n+a+b+c)^{2}(n+a+b+c+1)}$ \\
\hline
\end{tabular}

Table 6: Limiting Expressions for the Posterior risks as $t \rightarrow \infty$ using the UP and the JP under PLF

\begin{tabular}{|c|c|c|}
\hline & \multicolumn{2}{|c|}{ Posterior Risks } \\
\hline Parameters & UP & JP \\
\hline$\beta_{1}$ & $\frac{2\left(n_{1}+1\right)}{\left(\sum_{k=1}^{n_{1}} x_{1 k}^{-1}\right)}\left\{\frac{\left(n_{1}+2\right)^{\frac{1}{2}}}{\left(n_{1}+1\right)^{\frac{1}{2}}}-1\right\}$ & $\frac{2 n_{1}}{\left(\sum_{k=1}^{n_{1}} x_{1 k}^{-1}\right)}\left\{\frac{\left(n_{1}+1\right)^{\frac{1}{2}}}{\left(n_{1}\right)^{\frac{1}{2}}}-1\right\}$ \\
\hline$\beta_{2}$ & $\frac{2\left(n_{2}+1\right)}{\left(\sum_{k=1}^{n_{2}} x_{2 k}^{-1}\right)}\left\{\frac{\left(n_{2}+2\right)^{\frac{1}{2}}}{\left(n_{2}+1\right)^{\frac{1}{2}}}-1\right\}$ & $\frac{2 n_{2}}{\left(\sum_{k=1}^{n_{2}} x_{2 k}^{-1}\right)}\left\{\frac{\left(n_{2}+1\right)^{\frac{1}{2}}}{\left(n_{2}\right)^{\frac{1}{2}}}-1\right\}$ \\
\hline$\beta_{3}$ & $\frac{2\left(n_{3}+1\right)}{\left(\sum_{k=1}^{n_{3}} x_{3 k}^{-1}\right)}\left\{\frac{\left(n_{3}+2\right)^{\frac{1}{2}}}{\left(n_{3}+1\right)^{\frac{1}{2}}}-1\right\}$ & $\frac{2 n_{3}}{\left(\sum_{k=1}^{n_{3}} x_{3 k}^{-1}\right)}\left\{\frac{\left(n_{3}+1\right)^{\frac{1}{2}}}{\left(n_{3}\right)^{\frac{1}{2}}}-1\right\}$ \\
\hline$p_{1}$ & $\frac{2\left(n_{1}+1\right)}{(n+3)}\left\{\frac{\left(\frac{\left(n_{1}+2\right)}{\left(n_{1}+1\right)}\right)^{\frac{1}{2}}}{\left(\frac{(n+4)}{(n+3)}\right)^{\frac{1}{2}}}-1\right\}$ & $\frac{2\left(n_{1}+1\right)}{(n+3)}\left\{\frac{\left(\frac{\left(n_{1}+2\right)}{\left(n_{1}+1\right)}\right)^{\frac{1}{2}}}{\left(\frac{(n+4)}{(n+3)}\right)^{\frac{1}{2}}}-1\right\}$ \\
\hline$p_{2}$ & $\frac{2\left(n_{2}+1\right)}{(n+3)}\left\{\frac{\left(\frac{\left.n_{2}+2\right)}{\left(n_{2}+1\right)}\right)^{\frac{1}{2}}}{\left(\frac{n+4)}{(n+3)}\right)^{\frac{1}{2}}}-1\right\}$ & $\frac{2\left(n_{2}+1\right)}{(n+3)}\left\{\frac{\left(\frac{\left.n_{2}+2\right)}{\left(n_{2}+1\right)}\right)^{\frac{1}{2}}}{\left(\frac{n+4)}{(n+3)}\right)^{\frac{1}{2}}}-1\right\}$ \\
\hline
\end{tabular}

Table 7: Limiting Expressions for the Posterior risks as $t \rightarrow \infty$ using the EP and ILP under PLF

\begin{tabular}{|c|c|c|}
\hline & \multicolumn{2}{|c|}{ Posterior Risks } \\
\hline Parameters & Exponential prior & Inverse Levy Prior \\
\hline$\beta_{1}$ & $\frac{2\left(n_{1}+1\right)}{\left(\sum_{k=1}^{n_{1}} x_{1 k}^{-1}+k_{1}\right)}\left\{\frac{\left(n_{1}+2\right)^{\frac{1}{2}}}{\left(n_{1}+1\right)^{\frac{1}{2}}}-1\right\}$ & $\frac{2\left(n_{1}+\frac{1}{2}\right)}{\left(\sum_{k=1}^{n_{1}} x_{1 k}^{-1}+\frac{a_{1}}{2}\right)}\left\{\frac{\left(n_{1}+\frac{3}{2}\right)^{\frac{1}{2}}}{\left(n_{1}+\frac{1}{2}\right)^{\frac{1}{2}}}-1\right\}$ \\
\hline$\beta_{2}$ & $\frac{2\left(n_{2}+1\right)}{\left(\sum_{k=1}^{n_{2}} x_{2 k}^{-1}+k_{2}\right)}\left\{\frac{\left(n_{2}+2\right)^{\frac{1}{2}}}{\left(n_{2}+1\right)^{\frac{1}{2}}}-1\right\}$ & $\frac{2\left(n_{2}+\frac{1}{2}\right)}{\left(\sum_{k=1}^{n_{2}} x_{2 k}^{-1}+\frac{a_{2}}{2}\right)}\left\{\frac{\left(n_{2}+\frac{3}{2}\right)^{\frac{1}{2}}}{\left(n_{2}+\frac{1}{2}\right)^{\frac{1}{2}}}-1\right\}$ \\
\hline$\beta_{3}$ & $\frac{2\left(n_{3}+1\right)}{\left(\sum_{k=1}^{n_{3}} x_{3 k}^{-1}+k_{3}\right)}\left\{\frac{\left(n_{3}+2\right)^{\frac{1}{2}}}{\left(n_{3}+1\right)^{\frac{1}{2}}}-1\right\}$ & $\frac{2\left(n_{3}+\frac{1}{2}\right)}{\left(\sum_{k=1}^{n_{3}} x_{3 k}^{-1}+\frac{a_{3}}{2}\right)}\left\{\frac{\left(n_{3}+\frac{3}{2}\right)^{\frac{1}{2}}}{\left(n_{3}+\frac{1}{2}\right)^{\frac{1}{2}}}-1\right\}$ \\
\hline$p_{1}$ & $\frac{2\left(n_{1}+a\right)}{(n+a+b+c)}\left\{\frac{\left(\frac{\left(n_{1}+a+1\right)}{\left(n_{1}+a\right)}\right)^{\frac{1}{2}}}{\left(\frac{(n+a+b+c+1}{(n+a+b+c)}\right)^{\frac{1}{2}}}-1\right\}$ & $\frac{2\left(n_{1}+a\right)}{(n+a+b+c)}\left\{\frac{\left(\frac{\left(n_{1}+a+1\right)}{\left.n_{1}+a\right)}\right)^{\frac{1}{2}}}{\left(\frac{(n+a+b+c+1)}{(n+a+b+c)}\right)^{\frac{1}{2}}}-1\right.$ \\
\hline$p_{2}$ & $\frac{2\left(n_{2}+b\right)}{(n+a+b+c)}\left\{\frac{\left(\frac{\left(n_{2}+b+1\right)}{\left(n_{1}+b\right)}\right)^{\frac{1}{2}}}{\left(\frac{(n+a+b+c+1)}{(n+a+b+c)}\right)^{\frac{1}{2}}}-1\right.$ & $\frac{2\left(n_{2}+b\right)}{(n+a+b+c)}\left\{\frac{\left(\frac{\left(n_{2}+b+1\right)}{(n+b)}\right)^{\frac{1}{2}}}{\left(\frac{(n+a+b+c+1)}{(n+a+b+c)}\right)^{\frac{1}{2}}}-1\right.$ \\
\hline
\end{tabular}




\section{Simulation Study}

A comprehensive simulation study was conducted in order to explore the performance of the Bayes estimators, impact of sample size and censoring rate to be appropriate for the model. Samples of sizes $n=25,40,55$ are generated from a 3-component mixture of the Frechet distributions with various set of the parametric values $\beta_{1}, \beta_{2}, \beta_{3}, p_{1}$ and $p_{2}$ fixed as $\left(\beta_{1}, \beta_{2}, \beta_{3}, p_{1}, p_{2}\right)=(0.50,1.0,1.50,0.30,0.50),(1.50,1.0,0.50,0.50,0.30)$. For fixed sample size, test termination time and set of parameters, the simulation is repeated 1000 times and the results are then averaged. Sample of sizes $p_{1} n, p_{2} n$ and $\left(1-p_{1}-p_{2}\right) n$ are chosen randomly from first component density $f_{1}\left(x ; \theta_{1}\right)$, second component density $f_{2}(x$; $\left.\theta_{2}\right)$ and third component density $f_{3}\left(x ; \theta_{3}\right)$, respectively.

Table 8: Limiting Expressions for the Bayes Posterior risks as $t \rightarrow \infty$ using the UP, the JP, the EP and the ILP under DLF

\begin{tabular}{|c|c|c|c|c|}
\hline & \multicolumn{4}{|c|}{ Posterior Risks } \\
\hline Parameters & UP & JP & EP & ILP \\
\hline$\beta_{1}$ & $\frac{n_{1}+1}{\left(n_{1}+1\right)\left(n_{1}+2\right)}$ & $\frac{n_{1}}{n_{1}\left(n_{1}+1\right)}$ & $\frac{n_{1}+1}{\left(n_{1}+1\right)\left(n_{1}+2\right)}$ & $\frac{n_{1}+0.5}{\left(n_{1}+0.5\right)\left(n_{1}+1\right)}$ \\
\hline$\beta_{2}$ & $\frac{n_{2}+1}{\left(n_{2}+1\right)\left(n_{2}+2\right)}$ & $\frac{n_{2}}{n_{2}\left(n_{2}+1\right)}$ & $\frac{n_{2}+1}{\left(n_{2}+1\right)\left(n_{2}+2\right)}$ & $\frac{n_{2}+0.5}{\left(n_{2}+0.5\right)\left(n_{2}+1\right)}$ \\
\hline$\beta_{3}$ & $\frac{n_{3}+1}{\left(n_{3}+1\right)\left(n_{3}+2\right)}$ & $\frac{n_{3}}{n_{3}\left(n_{3}+1\right)}$ & $\frac{n_{3}+1}{\left(n_{3}+1\right)\left(n_{3}+2\right)}$ & $1-\frac{\left(n_{1}+a\right)(n+a+b+c)}{\left(n_{1}+a+1\right)(n+a+b+c)}$ \\
\hline$p_{1}$ & $1-\frac{\left(n_{1}+1\right)(n+4)}{\left(n_{1}+2\right)(n+3)}$ & $1-\frac{\left(n_{1}+1\right)(n+4)}{\left(n_{1}+2\right)(n+3)}$ & $1-\frac{\left(n_{1}+a\right)(n+a+b+c+1)}{\left(n_{1}+a+1\right)(n+a+b+c)}$ & $1-\frac{\left(n_{2}+b\right)(n+a+b+c+1)}{\left(n_{2}+b+1\right)(n+a+b+c)}$ \\
\hline$p_{2}$ & $1-\frac{\left(n_{2}+1\right)(n+4)}{\left(n_{2}+2\right)(n+3)}$ & $1-\frac{\left(n_{2}+1\right)(n+4)}{\left(n_{2}+2\right)(n+3)}$ & $1-\frac{\left(n_{2}+b\right)(n+a+b+c+1)}{\left(n_{2}+b+1\right)(n+a+b+c)}$ & 1 \\
\hline
\end{tabular}

The observations which are greater than a fixed $t$ are declared as censored observations. For each $t$ only failures have been inspected either as a member of subpopulation-I or subpopulation-II or subpopulation-III. On the basis of each sample size, the Bayes estimators (BEs) and Posterior risks (PRs) are computed using the informative and noninformative priors under SELF, PLF and DLF. In order to conduct Bayesian analysis under informative priors, elicitation of hyper-parameters is obtained by using the prior predictive approach. In order to evaluate the impact of test termination time on Bayes estimators, the Type-I right censoring scheme is used for fixed test termination time $t=15$ and 20. For each of the 1000 samples, the Bayes estimators and Posterior risks were calculated using a routine in Mathematica 10.0 and the results are presented in Tables 916. The simulation study gives us some interesting characteristics of the Bayes estimates. The properties have been foregrounded in terms of sample sizes, size of mixing proportion parameters, different loss functions and censoring rates. It is noticed that because of censoring, the posterior risks of all the parameters are reduced with an increase in sample size.

\section{A Real Life Data Application}

Crowder et al. (1994) reported the data on fiber failure strength. The breaking strength of fiber section of lengths 5, 12, 30 and $45 \mathrm{~mm}$. To elucidate the proposed methodology, we take the data on 3-component, namely 5, 12 and 30mm, respectively. The values are right 
censored at 4.0 i.e. $\mathrm{t}=4.0$. The sample statistics required to evaluate the proposed estimates are as follows:

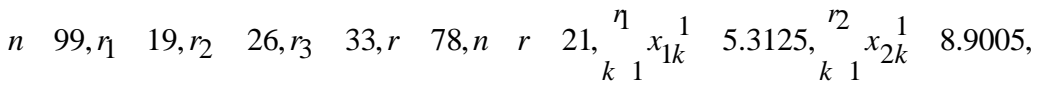

$$
\begin{aligned}
& \underset{k \square 1}{\prod_{13}} x_{3 k}^{\square 1} \square 13.5522 .
\end{aligned}
$$

Bayes estimates and Bayes Posterior risks using the UP, the JP, the EP and the ILP under SELF, PLF and DLF are in Table 17 given in appendix.

It is noted that the results gained from real data are compatible with simulation results. The results declare that the execution of the informative prior is better than the noninformative priors. It is also examined that execution of DLF preferred for estimating the component parameters, while SELF better for estimating the proportion parameters.

\section{Final Remarks}

In this study, the Bayesian estimation of 3-component mixture of the Frechet distributions has been considered assuming the case when the shape parameter is known based on type-I censored data. The purpose of this paper is to find out the appropriate combinations of prior distributions and loss functions to estimate the parameters of the 3component mixture of the Frechet distributions. We conducted all-encompassing simulation study to find out the relative performance of the Bayes estimators when the shape parameter is assumed to be known. From simulated results, we observed that an increase in the sample size and test termination time provides better Bayes estimators. Furthermore, as sample size increases (decreases) the posterior risks of Bayes estimators' decreases (increases) for a fixed test termination time. Also, the DLF is perceived as an appropriate choice for estimating component parameters and SELF is expedient for estimating the proportion parameters. Finally, we deduce that the EP is apt prior in order to estimate the component parameters. When SELF is used, the EP is an appropriate prior for proportion parameters. The similar pattern is examined for the JP when noninformative priors are contemplated.

\section{References}

1. Abbas, K. and Y. Tang. Analysis of Frechet Distribution Using Reference Priors, Communications in Statistics-Theory and Methods 44(14), 2945-2956, 2015.

2. Abbas, K. and T. Yincai. Comparison of Estimation Methods for Frechet Distribution with Known Shape, Caspian Journal of Applied Sciences Research 1 (10), 2012.

3. Ali, S. Mixture of the inverse Rayleigh distribution: Properties and estimation in a Bayesian framework, Applied Mathematical Modelling 39(2), 515-530, 2015.

4. Anwar, S., Shahab, S., and Islam, A.U. Accelerated Life Testing Design Using Geometric Process for Marshall-Olkin Extended Exponential Distribution with Type I Censored Data, International Journal of Technology Enhancements and Emerging Engineering Research 3(1), 179186, 2014. 
5. Aslam, M. An application of prior predictive distribution to elicit the prior density, Journal of Statistical Theory and applications 2(1), 70-83, 2003.

6. Aslam, M., Tahir, M., Hussain, Z., \& Al-Zahrani, B. A 3-Component Mixture of Rayleigh Distributions: Properties and Estimation in Bayesian Framework, PloS one 10(5), e0126183, 2015.

7. Chatterjee, A., and Chatterjee, A. Use of the Frechet distribution for UPV measurements in concrete, NDT and E International 52, 122-128, 2012

8. Crowder, M. J., A. Kimber, et al. Statistical analysis of reliability data (CRC Press, 1994).

9. DeGroot, M. H. Optimal statistical decisions (John Wiley and Sons, 2005).

10. Frechet, M. Sur la loi de probabilite de lecart maximum, Annales Soc.Polon. Math 6(95), 1927.

11. Gijbels, I. (2010). Censored data, Wiley Interdisciplinary Reviews: Computational Statistics 2(2), 178-188, 2010.

12. Harlow, D. G. Applications of the Frechet distribution function, International Journal of Materials and Product Technology 17(5-6), 482-495, 2002.

13. Jeffreys, H. An invariant form for the prior probability in estimation problems, Proceedings of the Royal Society of London A: Mathematical, Physical and Engineering Sciences, The Royal Society, 1946.

14. Jeffreys, H. The theory of probability (OUP Oxford, 1998).

15. Kalbfleisch, J. D. and R. L. Prentice. The statistical analysis of failure time data (John Wiley \& Sons, 2011).

16. Kundu, D. and H. Howlader. Bayesian inference and prediction of the inverse Weibull distribution for Type-II censored data, Computational Statistics and Data Analysis 54, 1547-1558, 2010.

17. Legendre, A. M. Nouvelles methodes pour la determination des orbites des cometes F. Didot, 1805.

18. Mendenhall, W. and R. Hader. Estimation of parameters of mixed exponentially distributed failure time distributions from censored life test data, Biometrika 45(3-4), 504-520, 1958.

19. Nadarajah, S. and S. Kotz. Sociological models based on Frechet random variables, Quality and Quantity 42 (1), 89-95, 2008.

20. Norstrom, J. G. The use of precautionary loss functions in risk analysis, IEEE Transactions on reliability 45 (3), 400-403, 1996.

21. Saleem, M., Aslam, M., \& Economou, P. On the Bayesian analysis of the mixture of power function distribution using the complete and the censored sample, Journal of Applied Statistics 37(1), 25-40, 2010.

22. Shi, Y. and Yan, W. The EB Estimation of Scale-parameter for Two Parameter Exponential Distribution Under the Type-I Censoring Life Test, 2010.

23. Zaharim, A., Najid, S. K., Razali, A.M., \& Sopian, K. Analyzing Malaysian wind speed data using statistical distribution, Proceedings of the 4th IASME/WSEAS International Conference on Energy and Environment, Cambridge, UK, 2009. 
Appendix

Table 9: Bayes estimates (BEs) and posterior risks (PRs) of 3-component mixture of Frechet distributions using the UP under SELF, PLF and DLF with $\beta_{1}=0.50, \beta_{2}=1.0, \beta_{3}=1.50, p_{1}=0.30, p_{2}=0.50$ and $t=15,20$

\begin{tabular}{|c|c|c|c|c|c|c|c|c|}
\hline \multirow[b]{2}{*}{$\mathrm{t}$} & \multirow[b]{2}{*}{$\mathrm{n}$} & \multirow{2}{*}{\multicolumn{2}{|c|}{ Loss Functions }} & \multicolumn{5}{|c|}{ UP } \\
\hline & & & & $\hat{\square_{1}}$ & $\hat{\square}_{2}$ & $\hat{D}_{3}$ & $\hat{p}_{1}$ & $\hat{p}_{2}$ \\
\hline \multirow{18}{*}{15} & \multirow{6}{*}{25} & \multirow{2}{*}{ SELF } & $\mathrm{BE}$ & 0.64377 & 1.15686 & 2.27870 & 0.32202 & 0.46107 \\
\hline & & & PR & 0.055320 & 0.117388 & 1.20838 & 0.007802 & 0.009023 \\
\hline & & \multirow{2}{*}{ PLF } & $\mathrm{BE}$ & 0.68530 & 1.22045 & 2.36739 & 0.33451 & 0.47026 \\
\hline & & & PR & 0.071868 & 0.092064 & 0.365849 & 0.023797 & 0.019441 \\
\hline & & \multirow{2}{*}{ DLF } & $\mathrm{BE}$ & 0.71542 & 1.26668 & 2.74792 & 0.34659 & 0.48023 \\
\hline & & & PR & 0.102186 & 0.074091 & 0.148938 & 0.070235 & 0.041193 \\
\hline & \multirow{6}{*}{40} & \multirow{2}{*}{ SELF } & $\mathrm{BE}$ & 0.59278 & 1.07921 & 1.89146 & 0.30288 & 0.48644 \\
\hline & & & PR & 0.030905 & 0.060258 & 0.501856 & 0.004986 & 0.006017 \\
\hline & & \multirow{2}{*}{ PLF } & $\mathrm{BE}$ & 0.61128 & 1.14019 & 2.03863 & 0.31102 & 0.49268 \\
\hline & & & PR & 0.045618 & 0.054225 & 0.220245 & 0.016255 & 0.012329 \\
\hline & & \multirow{2}{*}{ DLF } & $\mathrm{BE}$ & 0.63787 & 1.14462 & 2.17595 & 0.31951 & 0.49756 \\
\hline & & & PR & 0.073250 & 0.047184 & 0.104961 & 0.051783 & 0.025131 \\
\hline & \multirow{6}{*}{55} & \multirow{2}{*}{ SELF } & $\mathrm{BE}$ & 0.56527 & 1.06674 & 1.82731 & 0.29273 & 0.49864 \\
\hline & & & PR & 0.020616 & 0.042147 & 0.32790 & 0.003668 & 0.004510 \\
\hline & & \multirow{2}{*}{ PLF } & $\mathrm{BE}$ & 0.58922 & 1.0999 & 1.87374 & 0.29925 & 0.50284 \\
\hline & & & PR & 0.034163 & 0.038209 & 0.154977 & 0.012369 & 0.009007 \\
\hline & & \multirow{2}{*}{ DLF } & $\mathrm{BE}$ & 0.59925 & 1.09831 & 2.0063 & 0.30568 & 0.50654 \\
\hline & & & PR & 0.057071 & 0.034554 & 0.080829 & 0.038614 & 0.016066 \\
\hline \multirow{18}{*}{20} & \multirow{6}{*}{25} & \multirow{2}{*}{ SELF } & $\mathrm{BE}$ & 0.63481 & 1.16243 & 2.29449 & 0.32138 & 0.46218 \\
\hline & & & PR & 0.053847 & 0.117997 & 1.2404 & 0.007732 & 0.008925 \\
\hline & & \multirow{2}{*}{ PLF } & $\mathrm{BE}$ & 0.61961 & 1.11891 & 2.01203 & 0.31088 & 0.49390 \\
\hline & & & PR & 0.045909 & 0.052604 & 0.214869 & 0.016092 & 0.012082 \\
\hline & & \multirow{2}{*}{ DLF } & $\mathrm{BE}$ & 0.71154 & 1.25977 & 2.65438 & 0.34577 & 0.48139 \\
\hline & & & PR & 0.101773 & $\mathbf{0 . 0 7 3 3 3 4}$ & 0.147498 & 0.069758 & 0.040334 \\
\hline & \multirow{6}{*}{40} & \multirow{2}{*}{ SELF } & $\mathrm{BE}$ & 0.58787 & 1.09944 & 1.93816 & 0.30233 & 0.48695 \\
\hline & & & PR & 0.029588 & 0.062360 & 0.509643 & 0.004938 & 0.005943 \\
\hline & & \multirow{2}{*}{ PLF } & $\mathrm{BE}$ & 0.58012 & 1.08996 & 1.86387 & 0.29923 & 0.50346 \\
\hline & & & PR & 0.033354 & $\mathbf{0 . 0 3 7 5 3 0}$ & 0.152627 & 0.012210 & 0.008854 \\
\hline & & \multirow{2}{*}{ DLF } & $\mathrm{BE}$ & 0.64474 & 1.15587 & 2.1441 & 0.31853 & 0.49929 \\
\hline & & & PR & 0.072878 & 0.046586 & 0.103819 & 0.051363 & 0.024471 \\
\hline & & SEI F & $\mathrm{BE}$ & 0.57336 & 1.06672 & 1.79499 & 0.29350 & 0.49884 \\
\hline & & SELF & PR & 0.021018 & $\mathbf{0 . 0 4 1 9 3 7}$ & $\mathbf{0 . 3 1 0 4 3 3}$ & 0.003621 & 0.004439 \\
\hline & 55 & PI F & $\mathrm{BE}$ & 0.56814 & 1.06748 & 1.83408 & 0.30622 & 0.49470 \\
\hline & 55 & PLF & PR & 0.025303 & 0.028511 & 0.120281 & 0.009811 & 0.007400 \\
\hline & & DIF & $\mathrm{BE}$ & 0.59795 & 1.11567 & 1.9722 & 0.30561 & 0.50807 \\
\hline & & DLF & PR & 0.056695 & 0.034157 & 0.080394 & 0.040446 & 0.017534 \\
\hline
\end{tabular}


Table 10: Bayes estimates (BEs) and posterior risks (PRs) of 3-component mixture of Frechet distributions using the UP under SELF, PLF and DLF with $\beta_{1}=1.50, \beta_{2}=1.0, \beta_{3}=0.50, p_{1}=0.50, p_{2}=0.30$ and $t=15,20$

\begin{tabular}{|c|c|c|c|c|c|c|c|c|}
\hline \multirow[b]{2}{*}{$\mathrm{t}$} & \multirow[b]{2}{*}{$\mathrm{n}$} & \multirow{2}{*}{\multicolumn{2}{|c|}{ Loss Functions }} & \multicolumn{5}{|c|}{ UP } \\
\hline & & & & $\hat{D}_{1}$ & $\hat{D}_{2}$ & $\hat{\square}_{3}$ & $\hat{p}_{1}$ & $\hat{p}_{2}$ \\
\hline \multirow{18}{*}{15} & \multirow{6}{*}{25} & \multirow{2}{*}{ SELF } & $\mathrm{BE}$ & 1.74859 & 1.29527 & 0.75497 & 0.46006 & 0.32346 \\
\hline & & & PR & 0.270075 & 0.225689 & 0.130652 & 0.009132 & 0.008040 \\
\hline & & \multirow{2}{*}{ PLF } & $\mathrm{BE}$ & 1.83555 & 1.35025 & 0.82037 & 0.47208 & 0.33400 \\
\hline & & & PR & 0.138592 & 0.14421 & 0.12496 & 0.01961 & 0.024479 \\
\hline & & \multirow{2}{*}{ DLF } & $\mathrm{BE}$ & 1.90234 & 1.44896 & 0.91036 & 0.48072 & 0.34776 \\
\hline & & & PR & 0.074547 & 0.104087 & 0.146394 & 0.041797 & 0.072502 \\
\hline & \multirow{6}{*}{40} & \multirow{2}{*}{ SELF } & $\mathrm{BE}$ & 1.65716 & 1.19814 & 0.65269 & 0.48610 & 0.30248 \\
\hline & & & PR & 0.143936 & 0.129094 & 0.056772 & 0.006090 & 0.005145 \\
\hline & & \multirow{2}{*}{ PLF } & $\mathrm{BE}$ & 1.70319 & 1.22146 & 0.67259 & 0.49308 & 0.31114 \\
\hline & & & PR & 0.081165 & 0.092916 & 0.070894 & 0.012431 & 0.016763 \\
\hline & & \multirow{2}{*}{ DLF } & $\mathrm{BE}$ & 1.72312 & 1.30093 & 0.73093 & 0.49883 & 0.32006 \\
\hline & & & PR & 0.047247 & 0.074745 & 0.102658 & 0.025266 & 0.053523 \\
\hline & \multirow{6}{*}{55} & \multirow{2}{*}{ SELF } & $\mathrm{BE}$ & 1.60162 & 1.14253 & 0.59521 & 0.49681 & 0.29308 \\
\hline & & & PR & 0.089013 & 0.086113 & 0.032680 & 0.003948 & 0.003298 \\
\hline & & \multirow{2}{*}{ PLF } & $\mathrm{BE}$ & 1.64833 & 1.17848 & 0.63863 & 0.50219 & 0.30031 \\
\hline & & & PR & 0.057169 & 0.069427 & 0.051312 & 0.009029 & 0.012717 \\
\hline & & \multirow{2}{*}{ DLF } & $\mathrm{BE}$ & 1.65163 & 1.21835 & 0.65325 & 0.50761 & 0.30658 \\
\hline & & & PR & 0.034042 & 0.058143 & 0.079678 & 0.017355 & 0.041676 \\
\hline \multirow{18}{*}{20} & \multirow{6}{*}{25} & \multirow{2}{*}{ SELF } & $\mathrm{BE}$ & 1.74164 & 1.26138 & 0.75824 & 0.46195 & 0.32211 \\
\hline & & & PR & 0.263732 & 0.2034 & 0.132482 & 0.008973 & 0.007877 \\
\hline & & \multirow{2}{*}{ PLF } & $\mathrm{BE}$ & 1.82546 & 1.34552 & 0.82642 & 0.47233 & 0.33397 \\
\hline & & & PR & 0.136515 & 0.141848 & 0.12488 & 0.019230 & 0.024031 \\
\hline & & \multirow{2}{*}{ DLF } & $\mathrm{BE}$ & 1.86253 & 1.45122 & 0.85807 & 0.48077 & 0.34732 \\
\hline & & & PR & 0.073767 & 0.102882 & 0.145164 & 0.040847 & 0.071070 \\
\hline & \multirow{6}{*}{40} & \multirow{2}{*}{ SELF } & $\mathrm{BE}$ & 1.66985 & 1.18325 & 0.65066 & 0.48769 & 0.30177 \\
\hline & & & PR & 0.143726 & 0.123538 & 0.056842 & 0.005962 & 0.005027 \\
\hline & & \multirow{2}{*}{ PLF } & $\mathrm{BE}$ & 1.68955 & 1.23244 & 0.69396 & 0.49364 & 0.31069 \\
\hline & & & PR & 0.079878 & 0.092856 & 0.072807 & 0.012222 & 0.016509 \\
\hline & & \multirow{2}{*}{ DLF } & $\mathrm{BE}$ & 1.72569 & 1.26653 & 0.71998 & 0.49894 & 0.31941 \\
\hline & & & PR & 0.046851 & 0.073979 & 0.101862 & 0.024806 & 0.052370 \\
\hline & & SEI F & $\mathrm{BE}$ & 1.60023 & 1.14286 & 0.60400 & 0.49943 & 0.29279 \\
\hline & & SELF & PR & 0.094254 & 0.085622 & 0.034621 & 0.004502 & 0.003697 \\
\hline & 55 & PIF & $\mathrm{BE}$ & 1.62303 & 1.1672 & 0.61780 & 0.50322 & 0.29982 \\
\hline & 5 & PLF & PR & 0.056158 & 0.067970 & 0.049419 & 0.008894 & 0.012476 \\
\hline & & DIF & $\mathrm{BE}$ & 1.65811 & 1.20219 & 0.65917 & 0.50655 & 0.30703 \\
\hline & & DLF & PR & 0.030623 & 0.057547 & 0.003026 & 0.002020 & 0.041193 \\
\hline
\end{tabular}


Table 11: Bayes estimates (BEs) and posterior risks (PRs) of 3-component mixture of Frechet distributions using the JP under SELF, PLF and DLF with $\beta_{1}=0.50, \beta_{2}=1.0, \beta_{3}=1.50, p_{1}=0.30, p_{2}=0.50$ and $t=15,20$

\begin{tabular}{|c|c|c|c|c|c|c|c|c|}
\hline \multirow[b]{2}{*}{$\mathrm{t}$} & \multirow[b]{2}{*}{$\mathrm{n}$} & \multirow{2}{*}{\multicolumn{2}{|c|}{ Loss Functions }} & \multicolumn{5}{|c|}{ JP } \\
\hline & & & & $\hat{D}_{1}$ & $\hat{D}_{2}$ & $\hat{\square}_{3}$ & $\hat{p}_{1}$ & $\hat{p}_{2}$ \\
\hline \multirow{18}{*}{15} & \multirow{6}{*}{25} & \multirow{2}{*}{ SELF } & $\mathrm{BE}$ & 0.58091 & 1.07457 & 1.85935 & 0.32217 & 0.46147 \\
\hline & & & PR & 0.051092 & 0.109223 & 0.951593 & 0.007807 & 0.009032 \\
\hline & & \multirow{2}{*}{ PLF } & $\mathrm{BE}$ & 0.60021 & 1.13312 & 2.08241 & 0.33307 & 0.47157 \\
\hline & & & PR & 0.070939 & 0.092292 & 0.381584 & 0.023884 & 0.019457 \\
\hline & & \multirow{2}{*}{ DLF } & $\mathrm{BE}$ & 0.65665 & 1.18461 & 2.34335 & 0.34626 & 0.48170 \\
\hline & & & PR & 0.114117 & 0.079845 & 0.176529 & 0.070452 & 0.041015 \\
\hline & \multirow{6}{*}{40} & \multirow{2}{*}{ SELF } & $\mathrm{BE}$ & 0.55630 & 1.05499 & 1.67388 & 0.30230 & 0.48745 \\
\hline & & & PR & 0.029581 & 0.060950 & 0.43985 & 0.004982 & 0.006022 \\
\hline & & \multirow{2}{*}{ PLF } & $\mathrm{BE}$ & 0.56735 & 1.077 & 1.83786 & 0.31062 & 0.49246 \\
\hline & & & PR & 0.045768 & 0.053800 & 0.221624 & 0.016264 & 0.012335 \\
\hline & & \multirow{2}{*}{ DLF } & $\mathrm{BE}$ & 0.59180 & 1.11057 & 1.95818 & 0.319287 & 0.49886 \\
\hline & & & PR & 0.079044 & 0.049363 & 0.117900 & 0.051770 & 0.024977 \\
\hline & \multirow{6}{*}{55} & \multirow{2}{*}{ SELF } & $\mathrm{BE}$ & 0.53448 & 1.02133 & 1.66279 & 0.29362 & 0.49907 \\
\hline & & & PR & 0.019683 & 0.039942 & 0.298843 & 0.003652 & 0.004498 \\
\hline & & \multirow{2}{*}{ PLF } & $\mathrm{BE}$ & 0.55075 & 1.04966 & 1.74973 & 0.29920 & 0.50327 \\
\hline & & & PR & 0.033769 & 0.037669 & 0.158084 & 0.012287 & 0.008977 \\
\hline & & \multirow{2}{*}{ DLF } & $\mathrm{BE}$ & 0.57305 & 1.07198 & 1.8412 & 0.30605 & 0.50608 \\
\hline & & & PR & 0.061325 & 0.036334 & 0.087157 & 0.041727 & 0.01864 \\
\hline \multirow{18}{*}{20} & \multirow{6}{*}{25} & \multirow{2}{*}{ SELF } & $\mathrm{BE}$ & 0.57603 & 1.07749 & 1.87336 & 0.32097 & 0.46305 \\
\hline & & & PR & 0.049727 & 0.109074 & 0.943486 & 0.007725 & 0.008929 \\
\hline & & \multirow{2}{*}{ PLF } & $\mathrm{BE}$ & 0.57162 & 1.08094 & 1.80660 & 0.31099 & 0.49397 \\
\hline & & & PR & 0.045693 & 0.053343 & 0.217134 & 0.016094 & 0.012096 \\
\hline & & \multirow{2}{*}{ DLF } & $\mathrm{BE}$ & 0.63484 & 1.16903 & 2.27552 & 0.34558 & 0.48200 \\
\hline & & & PR & 0.113312 & 0.079047 & 0.173688 & 0.069736 & 0.040230 \\
\hline & \multirow{6}{*}{40} & \multirow{2}{*}{ SELF } & $\mathrm{BE}$ & 0.54322 & 1.0624 & 1.73359 & 0.30293 & 0.48638 \\
\hline & & & PR & 0.027587 & 0.061416 & 0.464869 & 0.004935 & 0.005931 \\
\hline & & \multirow{2}{*}{ PLF } & $\mathrm{BE}$ & 0.55228 & 1.05684 & 1.72704 & 0.29962 & 0.50377 \\
\hline & & & PR & 0.033652 & 0.037662 & 0.154077 & 0.012207 & 0.008851 \\
\hline & & \multirow{2}{*}{ DLF } & $\mathrm{BE}$ & 0.58417 & 1.10718 & 1.91391 & 0.31895 & 0.49892 \\
\hline & & & PR & 0.078584 & 0.048943 & 0.116287 & $\mathbf{0 . 0 5 1 2 9 7}$ & 0.024487 \\
\hline & & SEI F & $\mathrm{BE}$ & 0.54156 & 1.02793 & 1.64473 & 0.29325 & 0.49877 \\
\hline & & SELF & PR & 0.020176 & 0.039843 & 0.283740 & 0.003626 & 0.004297 \\
\hline & 55 & PI F & $\mathrm{BE}$ & 0.53905 & 1.03189 & 1.67272 & 0.30641 & 0.49593 \\
\hline & כנ & PLF & PR & 0.025535 & 0.029513 & 0.11985 & 0.009916 & 0.006883 \\
\hline & & DIF & $\mathrm{BE}$ & 0.56019 & 1.07439 & 1.82138 & 0.30513 & 0.50741 \\
\hline & & DLF & PR & 0.060170 & 0.035413 & 0.086891 & 0.040516 & 0.017614 \\
\hline
\end{tabular}


Table 12: Bayes estimates (BEs) and posterior risks (PRs) of 3-component mixture of Frechet distributions using the JP under SELF, PLF and DLF with $\beta_{1}=1.50, \beta_{2}=1.0, \beta_{3}=0.50, p_{1}=0.50, p_{2}=0.30$ and $t=15,20$

\begin{tabular}{|c|c|c|c|c|c|c|c|c|}
\hline \multirow[b]{2}{*}{$\mathrm{t}$} & \multirow[b]{2}{*}{$\mathrm{n}$} & \multirow{2}{*}{\multicolumn{2}{|c|}{ Loss Functions }} & \multicolumn{5}{|c|}{ JP } \\
\hline & & & & $\hat{\square}_{1}$ & $\hat{D_{2}}$ & $\hat{\square}_{3}$ & $\hat{p}_{1}$ & $\hat{p}_{2}$ \\
\hline \multirow{18}{*}{15} & \multirow{6}{*}{25} & \multirow{2}{*}{ SELF } & $\mathrm{BE}$ & 1.62451 & 1.15324 & 0.61133 & 0.46153 & 0.32283 \\
\hline & & & PR & 0.252026 & 0.201082 & 0.108391 & 0.009131 & 0.008023 \\
\hline & & \multirow{2}{*}{ PLF } & $\mathrm{BE}$ & 1.70086 & 1.21865 & 0.69166 & 0.47090 & 0.33565 \\
\hline & & & PR & 0.139487 & 0.145222 & 0.124433 & 0.019688 & 0.024467 \\
\hline & & \multirow{2}{*}{ DLF } & $\mathrm{BE}$ & 1.77249 & 1.3088 & 0.75414 & 0.48225 & 0.34643 \\
\hline & & & PR & 0.080069 & 0.116407 & 0.171114 & 0.041300 & 0.072567 \\
\hline & \multirow{6}{*}{40} & \multirow{2}{*}{ SELF } & $\mathrm{BE}$ & 1.58907 & 1.1068 & 0.58480 & 0.48657 & 0.30299 \\
\hline & & & PR & 0.139066 & 0.118858 & 0.051264 & 0.006084 & 0.005144 \\
\hline & & \multirow{2}{*}{ PLF } & $\mathrm{BE}$ & 1.61493 & 1.14689 & 0.61894 & 0.49342 & 0.31113 \\
\hline & & & PR & 0.080890 & 0.094580 & 0.073166 & 0.012458 & 0.0167985 \\
\hline & & \multirow{2}{*}{ DLF } & $\mathrm{BE}$ & 1.64244 & 1.18336 & 0.66932 & 0.49876 & 0.32040 \\
\hline & & & PR & 0.049582 & 0.080615 & 0.114455 & 0.025244 & $\mathbf{0 . 0 5 3 3 3 0}$ \\
\hline & \multirow{6}{*}{55} & \multirow{2}{*}{ SELF } & $\mathrm{BE}$ & 1.54534 & 1.08178 & 0.54608 & 0.49890 & 0.29365 \\
\hline & & & PR & 0.093020 & 0.080521 & 0.031340 & 0.002569 & 0.003754 \\
\hline & & \multirow{2}{*}{ PLF } & $\mathrm{BE}$ & 1.58687 & 1.10878 & 0.57196 & 0.50317 & 0.30007 \\
\hline & & & PR & 0.057264 & 0.069425 & 0.050113 & 0.009072 & 0.012764 \\
\hline & & \multirow{2}{*}{ DLF } & $\mathrm{BE}$ & 1.58564 & 1.14535 & 0.60851 & 0.50466 & 0.30500 \\
\hline & & & PR & 0.036722 & 0.056727 & 0.085868 & 0.018976 & 0.042790 \\
\hline \multirow{18}{*}{20} & \multirow{6}{*}{25} & \multirow{2}{*}{ SELF } & $\mathrm{BE}$ & 1.62234 & 1.15064 & 0.60662 & 0.46212 & 0.32254 \\
\hline & & & PR & 0.249683 & 0.199318 & 0.098794 & 0.008987 & 0.007898 \\
\hline & & \multirow{2}{*}{ PLF } & $\mathrm{BE}$ & 1.71172 & 1.2034 & 0.68101 & 0.47208 & 0.33446 \\
\hline & & & PR & 0.138772 & 0.142427 & 0.121547 & 0.019336 & 0.024137 \\
\hline & & \multirow{2}{*}{ DLF } & $\mathrm{BE}$ & 1.78693 & 1.33034 & 0.76499 & 0.48152 & 0.34689 \\
\hline & & & PR & 0.079564 & 0.114999 & 0.170062 & 0.040795 & 0.071248 \\
\hline & \multirow{6}{*}{40} & \multirow{2}{*}{ SELF } & $\mathrm{BE}$ & 1.58633 & 1.08764 & 0.57886 & 0.48664 & 0.30311 \\
\hline & & & PR & 0.136908 & 0.112561 & 0.049910 & 0.005969 & 0.005047 \\
\hline & & \multirow{2}{*}{ PLF } & $\mathrm{BE}$ & 1.6213 & 1.14751 & 0.60402 & 0.49260 & 0.31195 \\
\hline & & & PR & 0.080508 & 0.093075 & 0.070809 & 0.012214 & 0.016453 \\
\hline & & \multirow{2}{*}{ DLF } & $\mathrm{BE}$ & 1.65336 & 1.19010 & 0.64195 & 0.49868 & 0.32036 \\
\hline & & & PR & 0.049082 & 0.079401 & 0.113552 & 0.024710 & 0.052125 \\
\hline & & SEI F & $\mathrm{BE}$ & 1.57172 & 1.05855 & 0.55101 & 0.49892 & 0.29354 \\
\hline & & SELF & PR & 0.093999 & 0.077687 & 0.031318 & 0.004417 & 0.003700 \\
\hline & 55 & PIF & $\mathrm{BE}$ & 1.58530 & 1.10819 & 0.57266 & 0.50527 & 0.29999 \\
\hline & 5 & PLF & PR & 0.065463 & 0.060803 & 0.039898 & 0.004309 & 0.009323 \\
\hline & & DIF & $\mathrm{BE}$ & 1.61441 & 1.12986 & 0.60430 & 0.50667 & 0.30620 \\
\hline & & DLF & PR & 0.037014 & 0.061531 & 0.085999 & 0.019852 & 0.038715 \\
\hline
\end{tabular}


Table 13: Bayes estimates (BEs) and posterior risks (PRs) of 3-component mixture of Frechet distributions using the EP under SELF, PLF and DLF with $\quad \square_{1} \square 0.50, \square_{2} \square 1.0, \square_{3} \square 1.50, k_{1} \square 2.0003, k_{2} \square 3.0030$, $k_{3} \square 4.0016, a \square 2.0103, b \square 1.7607, c \square 1.50, p_{1} \square 0.30, p_{2} \square 0.50, t \square 15,20$

\begin{tabular}{|c|c|c|c|c|c|c|c|c|}
\hline \multirow[b]{2}{*}{$\mathrm{t}$} & \multirow[b]{2}{*}{$\mathrm{n}$} & \multirow{2}{*}{\multicolumn{2}{|c|}{ Loss Functions }} & \multicolumn{5}{|c|}{$\mathrm{EP}$} \\
\hline & & & & $\hat{D}_{1}$ & $\hat{D}_{2}$ & $\hat{D}_{3}$ & $\hat{p}_{1}$ & $\hat{p}_{2}$ \\
\hline \multirow{18}{*}{15} & \multirow{6}{*}{25} & \multirow{2}{*}{ SELF } & $\mathrm{BE}$ & 0.55551 & 0.92274 & 0.82568 & 0.33441 & 0.45521 \\
\hline & & & PR & 0.038928 & 0.071285 & 0.126697 & 0.007427 & 0.008348 \\
\hline & & \multirow{2}{*}{ PLF } & $\mathrm{BE}$ & 0.60325 & 0.96364 & 0.88972 & 0.34532 & 0.46587 \\
\hline & & & PR & 0.063094 & 0.071811 & 0.142077 & 0.021865 & 0.018126 \\
\hline & & \multirow{2}{*}{ DLF } & $\mathrm{BE}$ & 0.61983 & 0.98655 & 0.98501 & 0.35626 & 0.47369 \\
\hline & & & PR & 0.101987 & 0.073424 & 0.152642 & 0.062567 & 0.038909 \\
\hline & \multirow{6}{*}{40} & \multirow{2}{*}{ SELF } & $\mathrm{BE}$ & 0.53918 & 0.94815 & 0.97882 & 0.31189 & 0.48209 \\
\hline & & & PR & 0.025079 & 0.045927 & 0.119074 & 0.004823 & 0.005667 \\
\hline & & \multirow{2}{*}{ PLF } & $\mathrm{BE}$ & 0.56027 & 0.97349 & 1.03919 & 0.31946 & 0.48807 \\
\hline & & & PR & 0.041728 & 0.045955 & 0.114565 & 0.015421 & 0.011869 \\
\hline & & \multirow{2}{*}{ DLF } & $\mathrm{BE}$ & 0.58569 & 0.99463 & 1.10532 & 0.32711 & 0.49421 \\
\hline & & & PR & 0.073225 & 0.046676 & 0.107356 & 0.047865 & 0.024225 \\
\hline & \multirow{6}{*}{55} & \multirow{2}{*}{ SELF } & $\mathrm{BE}$ & 0.53334 & 0.96646 & 1.07127 & 0.29926 & 0.49509 \\
\hline & & & PR & 0.018262 & 0.033959 & 0.106453 & 0.001430 & 0.003336 \\
\hline & & \multirow{2}{*}{ PLF } & $\mathrm{BE}$ & 0.54891 & 0.98020 & 1.13557 & 0.30700 & 0.49951 \\
\hline & & & PR & 0.031655 & $\mathbf{0 . 0 3 3 7 3 5}$ & 0.095852 & 0.015732 & 0.010110 \\
\hline & & \multirow{2}{*}{ DLF } & $\mathrm{BE}$ & 0.57275 & 0.99541 & 1.18032 & 0.31050 & 0.50200 \\
\hline & & & PR & 0.056119 & 0.041438 & 0.083530 & 0.673658 & 0.241394 \\
\hline \multirow{18}{*}{20} & \multirow{6}{*}{25} & \multirow{2}{*}{ SELF } & $\mathrm{BE}$ & 0.56097 & 0.92326 & 0.84294 & 0.33325 & 0.45548 \\
\hline & & & PR & 0.039252 & $\mathbf{0 . 0 7 0 5 3 1}$ & 0.129385 & 0.007329 & 0.008234 \\
\hline & & \multirow{2}{*}{ PLF } & $\mathrm{BE}$ & 0.59266 & 0.95149 & 0.90292 & 0.34441 & 0.46431 \\
\hline & & & PR & 0.061654 & 0.070581 & 0.140858 & 0.021652 & 0.017932 \\
\hline & & \multirow{2}{*}{ DLF } & $\mathrm{BE}$ & 0.62771 & 0.99097 & 0.97710 & 0.35541 & 0.47325 \\
\hline & & & PR & 0.10156 & 0.072974 & 0.150129 & 0.062167 & 0.038447 \\
\hline & \multirow{6}{*}{40} & \multirow{2}{*}{ SELF } & $\mathrm{BE}$ & 0.53557 & 0.95070 & 0.98197 & 0.31147 & 0.48189 \\
\hline & & & PR & 0.024145 & 0.045846 & 0.118041 & 0.004779 & 0.005613 \\
\hline & & \multirow{2}{*}{ PLF } & $\mathrm{BE}$ & 0.56062 & 0.98039 & 1.03043 & 0.31939 & 0.48731 \\
\hline & & & PR & 0.041432 & 0.046058 & 0.111838 & 0.015184 & 0.011632 \\
\hline & & \multirow{2}{*}{ DLF } & $\mathrm{BE}$ & 0.58082 & 0.99449 & 1.07534 & 0.32699 & 0.49348 \\
\hline & & & PR & 0.072586 & 0.046353 & 0.105614 & 0.047042 & 0.023690 \\
\hline & & SEI F & $\mathrm{BE}$ & 0.52819 & 0.95968 & 1.08422 & 0.29886 & 0.49330 \\
\hline & & SELF & PR & 0.017760 & $\mathbf{0 . 0 3 3 2 7 9}$ & 0.107704 & 0.003396 & 0.004097 \\
\hline & 55 & PI F & $\mathrm{BE}$ & 0.54724 & 0.96713 & 1.12129 & 0.30603 & 0.49888 \\
\hline & כ5 & PLF & PR & 0.031294 & $\mathbf{0 . 0 3 3 0 6 3}$ & 0.092939 & 0.016503 & 0.015029 \\
\hline & & DLF & $\mathrm{BE}$ & 0.56154 & 0.99369 & 1.18479 & 0.31140 & 0.50137 \\
\hline & & DLF & PR & 0.056394 & $\mathbf{0 . 0 3 3 9 1 7}$ & 0.080692 & 0.114385 & 0.010104 \\
\hline
\end{tabular}


Table 14: Bayes estimates (BEs) and posterior risks (PRs) of 3-component mixture of Frechet distributions using the EP under SELF, PLF and DLF with $\square_{1} \square 1.50, \square_{2} \square 1.0, \square_{3} \square 0.50, k_{1} \square 2.0003, k_{2} \square 3.0030, k_{3} \square 4.0016, a \square 2.0103$, $b \square 1.7607, c \square 1.50, p_{1} \square 0.50, p_{2} \square 0.30, t \square 15,20$.

\begin{tabular}{|c|c|c|c|c|c|c|c|c|}
\hline \multirow[b]{2}{*}{$\mathrm{t}$} & \multirow[b]{2}{*}{$\mathrm{n}$} & \multirow{2}{*}{\multicolumn{2}{|c|}{ Loss Functions }} & \multicolumn{5}{|c|}{ EP } \\
\hline & & & & $\hat{D}_{1}$ & $\hat{\square}_{2}$ & $\hat{D}_{3}$ & $\hat{p}_{1}$ & $\hat{p}_{2}$ \\
\hline \multirow{18}{*}{15} & \multirow{6}{*}{25} & \multirow{2}{*}{ SELF } & $\mathrm{BE}$ & 1.36721 & 0.85140 & 0.46772 & 0.46301 & 0.32171 \\
\hline & & & PR & 0.157862 & 0.089797 & 0.041536 & 0.008446 & 0.007398 \\
\hline & & \multirow{2}{*}{ PLF } & $\mathrm{BE}$ & 1.4189 & 0.92149 & 0.50909 & 0.47024 & 0.33399 \\
\hline & & & PR & 0.107054 & 0.098496 & 0.077229 & 0.018129 & 0.022563 \\
\hline & & \multirow{2}{*}{ DLF } & $\mathrm{BE}$ & 1.47634 & 0.97137 & 0.56335 & 0.48053 & 0.34477 \\
\hline & & & PR & 0.073811 & 0.10423 & 0.146148 & 0.038094 & 0.066913 \\
\hline & \multirow{6}{*}{40} & \multirow{2}{*}{ SELF } & $\mathrm{BE}$ & 1.40294 & 0.93721 & 0.48385 & 0.48530 & 0.30402 \\
\hline & & & PR & 0.101245 & 0.075063 & 0.029030 & 0.005763 & 0.004883 \\
\hline & & \multirow{2}{*}{ PLF } & $\mathrm{BE}$ & 1.43686 & 0.95059 & 0.52373 & 0.49043 & 0.31218 \\
\hline & & & PR & 0.068436 & 0.072228 & 0.054999 & 0.011804 & 0.015799 \\
\hline & & \multirow{2}{*}{ DLF } & $\mathrm{BE}$ & 1.49100 & 0.99448 & 0.54173 & 0.49686 & 0.32017 \\
\hline & & & PR & 0.047121 & 0.074741 & 0.102588 & 0.023844 & 0.050124 \\
\hline & \multirow{6}{*}{55} & \multirow{2}{*}{ SELF } & $\mathrm{BE}$ & 1.43967 & 0.92606 & 0.50277 & 0.49547 & 0.29381 \\
\hline & & & PR & 0.076162 & 0.055728 & 0.023097 & 0.004015 & 0.003477 \\
\hline & & \multirow{2}{*}{ PLF } & $\mathrm{BE}$ & 1.47659 & 0.96460 & 0.50801 & 0.50167 & 0.30105 \\
\hline & & & PR & 0.051274 & 0.056849 & 0.041014 & 0.010081 & 0.013542 \\
\hline & & \multirow{2}{*}{ DLF } & $\mathrm{BE}$ & 1.49007 & 1.00929 & 0.54053 & 0.49697 & 0.30087 \\
\hline & & & PR & 0.034447 & 0.058223 & 0.079346 & 0.019807 & 0.036419 \\
\hline \multirow{18}{*}{20} & \multirow{6}{*}{25} & \multirow{2}{*}{ SELF } & $\mathrm{BE}$ & 1.3559 & 0.88419 & 0.47244 & 0.46168 & 0.32347 \\
\hline & & & PR & 0.154326 & 0.095135 & 0.042023 & 0.008297 & 0.007299 \\
\hline & & \multirow{2}{*}{ PLF } & $\mathrm{BE}$ & 1.42931 & 0.91622 & 0.51409 & 0.47185 & 0.33300 \\
\hline & & & PR & 0.106651 & 0.097322 & 0.077748 & 0.017816 & $\mathbf{0 . 0 2 2 2 9 0}$ \\
\hline & & \multirow{2}{*}{ DLF } & $\mathrm{BE}$ & 1.48645 & 0.97129 & 0.54757 & 0.47943 & 0.34577 \\
\hline & & & PR & 0.073451 & 0.102901 & 0.145475 & 0.037700 & 0.065653 \\
\hline & \multirow{6}{*}{40} & \multirow{2}{*}{ SELF } & $\mathrm{BE}$ & 1.4347 & 0.91166 & 0.48972 & 0.48624 & 0.30334 \\
\hline & & & PR & 0.104301 & 0.070267 & 0.029489 & 0.005681 & 0.004792 \\
\hline & & \multirow{2}{*}{ PLF } & $\mathrm{BE}$ & 1.45956 & 0.94762 & 0.51860 & 0.49190 & 0.31141 \\
\hline & & & PR & 0.068724 & 0.071368 & 0.054277 & 0.011574 & 0.015574 \\
\hline & & \multirow{2}{*}{ DLF } & $\mathrm{BE}$ & 1.49852 & 0.99278 & 0.53416 & 0.49705 & 0.31990 \\
\hline & & & PR & 0.046634 & 0.073803 & 0.101863 & 0.023495 & 0.049426 \\
\hline & & SEI F & $\mathrm{BE}$ & 1.43816 & 0.93488 & 0.50274 & 0.49735 & 0.29321 \\
\hline & & & PR & 0.075478 & 0.056123 & 0.023135 & 0.003680 & 0.003524 \\
\hline & 55 & PIF & $\mathrm{BE}$ & 1.45763 & 0.95834 & 0.51495 & 0.50311 & 0.30110 \\
\hline & & & PR & 0.047920 & 0.055543 & 0.041162 & 0.002523 & 0.000927 \\
\hline & & DI F & $\mathrm{BE}$ & 1.48625 & 0.99030 & 0.53579 & 0.50456 & 0.30614 \\
\hline & & DLF & PR & 0.034065 & 0.057383 & 0.078288 & 0.012819 & 0.021566 \\
\hline
\end{tabular}


Table 15: Bayes estimates (BEs) and posterior risks (PRs) of 3-component mixture of Frechet distributions using the ILP under SELF, PLF and DLF with $\square_{1} \square 0.50, \square_{2} \square 1.0, \square_{3} \square 1.50, a_{1} \square 1.9520, a_{2} \square 2.5321, a_{3} \square 3.7735, a \square 0.2763$, $b \square 0.1167, c \square 1.0, p_{1} \square 0.30, p_{2} \square 0.50, t \square 15,20$.

\begin{tabular}{|c|c|c|c|c|c|c|c|c|}
\hline \multirow[b]{2}{*}{$\mathrm{t}$} & \multirow[b]{2}{*}{$\mathrm{n}$} & \multirow{2}{*}{\multicolumn{2}{|c|}{ Loss Functions }} & \multicolumn{5}{|c|}{ ILP } \\
\hline & & & & $\hat{D}_{1}$ & $\hat{\square}_{2}$ & $\hat{\square}_{3}$ & $\hat{p}_{1}$ & $\hat{p}_{2}$ \\
\hline \multirow{18}{*}{15} & \multirow{6}{*}{25} & \multirow{2}{*}{ SELF } & $\mathrm{BE}$ & 0.56523 & 1.01139 & 1.12826 & 0.31465 & 0.45912 \\
\hline & & & PR & 0.044554 & 0.090911 & 0.267451 & $\mathbf{0 . 0 0 8 2 3 0}$ & 0.009621 \\
\hline & & \multirow{2}{*}{ PLF } & $\mathrm{BE}$ & 0.59757 & 1.04628 & 1.23254 & 0.32907 & 0.46956 \\
\hline & & & PR & 0.065972 & 0.081507 & 0.211980 & 0.025600 & 0.020770 \\
\hline & & \multirow{2}{*}{ DLF } & $\mathrm{BE}$ & 0.63355 & 1.0946 & 1.33595 & 0.34240 & 0.47914 \\
\hline & & & PR & 0.107596 & 0.076576 & 0.164423 & 0.076680 & 0.044163 \\
\hline & \multirow{6}{*}{40} & \multirow{2}{*}{ SELF } & $\mathrm{BE}$ & 0.54201 & 1.00966 & 1.23515 & 0.29845 & 0.48646 \\
\hline & & & PR & 0.026295 & $\mathbf{0 . 0 5 3 8 7 3}$ & 0.208363 & 0.005167 & 0.006284 \\
\hline & & \multirow[b]{2}{*}{ PLF } & $\mathrm{BE}$ & 0.57394 & 1.02998 & 1.31238 & 0.30605 & 0.49318 \\
\hline & & & PR & 0.044519 & 0.049916 & 0.152275 & 0.017071 & 0.012805 \\
\hline & & \multirow{2}{*}{ DLF } & $\mathrm{BE}$ & 0.59161 & 1.06348 & 1.40867 & 0.31462 & 0.49928 \\
\hline & & & PR & 0.076175 & 0.047963 & 0.112568 & 0.055395 & 0.026073 \\
\hline & \multirow{6}{*}{55} & \multirow{2}{*}{ SELF } & $\mathrm{BE}$ & 0.53336 & 1.00855 & 1.30808 & 0.28957 & 0.49727 \\
\hline & & & PR & 0.018927 & 0.038060 & 0.169564 & $\mathbf{0 . 0 0 3 8 3 8}$ & 0.004994 \\
\hline & & \multirow{2}{*}{ PLF } & $\mathrm{BE}$ & 0.55034 & 1.03025 & 1.38071 & 0.29622 & 0.50312 \\
\hline & & & PR & 0.032747 & 0.036346 & 0.121035 & 0.015682 & 0.014800 \\
\hline & & \multirow{2}{*}{ DLF } & $\mathrm{BE}$ & 0.57377 & 1.04171 & 1.42649 & 0.30118 & 0.50569 \\
\hline & & & PR & 0.058687 & 0.034896 & 0.085874 & 0.044595 & 0.019809 \\
\hline \multirow{18}{*}{20} & \multirow{6}{*}{25} & \multirow{2}{*}{ SELF } & $\mathrm{BE}$ & 0.57062 & 1.00137 & 1.12997 & 0.31516 & 0.45869 \\
\hline & & & PR & 0.044086 & 0.087920 & 0.263408 & 0.008132 & 0.009446 \\
\hline & & \multirow{2}{*}{ PLF } & $\mathrm{BE}$ & 0.59099 & 1.04844 & 1.23516 & 0.32663 & 0.47035 \\
\hline & & & PR & 0.065263 & 0.080717 & 0.208417 & 0.025358 & 0.020369 \\
\hline & & \multirow{2}{*}{ DLF } & $\mathrm{BE}$ & 0.63333 & 1.08936 & 1.32068 & 0.34144 & 0.47984 \\
\hline & & & PR & 0.10689 & $\mathbf{0 . 0 7 5 7 3 0}$ & 0.161844 & $\mathbf{0 . 0 7 5 8 4 7}$ & 0.043153 \\
\hline & \multirow{6}{*}{40} & \multirow{2}{*}{ SELF } & $\mathrm{BE}$ & 0.54405 & 1.00478 & 1.24465 & 0.29727 & 0.48533 \\
\hline & & & PR & 0.026580 & 0.053027 & 0.20577 & 0.005089 & 0.006168 \\
\hline & & \multirow{2}{*}{ PLF } & $\mathrm{BE}$ & 0.56536 & 1.02789 & 1.33523 & 0.30662 & 0.49226 \\
\hline & & & PR & 0.043443 & 0.049528 & 0.152538 & 0.016898 & 0.012658 \\
\hline & & \multirow{2}{*}{ DLF } & $\mathrm{BE}$ & 0.5856 & 1.05776 & 1.41234 & 0.31451 & 0.49793 \\
\hline & & & PR & 0.075514 & 0.047664 & 0.110136 & 0.054559 & 0.025622 \\
\hline & & SEI F & $\mathrm{BE}$ & 0.53292 & 1.0021 & 1.31814 & 0.28968 & 0.49703 \\
\hline & & SELF & PR & 0.018825 & 0.037525 & 0.168365 & 0.003640 & 0.004276 \\
\hline & 55 & PI F & $\mathrm{BE}$ & 0.54706 & 1.02466 & 1.37435 & 0.29611 & 0.50310 \\
\hline & נת & PLГ & PR & 0.032300 & $\mathbf{0 . 0 3 5 8 3 5}$ & 0.118065 & 0.012781 & 0.008715 \\
\hline & & DIF & $\mathrm{BE}$ & 0.56482 & 1.03511 & 1.43552 & 0.29579 & 0.48353 \\
\hline & & DLF & PR & 0.058186 & 0.034708 & 0.084337 & 0.040373 & 0.016322 \\
\hline
\end{tabular}


Table 16: Bayes estimates (BEs) and posterior risks (PRs) of 3-component mixture of Frechet distributions using the ILP under SELF, PLF and DLF with $\square_{1} \square 1.50, \square_{2} \square 1.0, \square_{3} \square 0.50, a_{1} \square 1.9520, a_{2} \square 2.5321, a_{3} \square 3.7735, a \square 0.2763$, $b \square 0.1167, c \square 1.0, p_{1} \square 0.50, p_{2} \square 0.30, t \square 15,20$.

\begin{tabular}{|c|c|c|c|c|c|c|c|c|}
\hline \multirow[b]{2}{*}{$\mathrm{t}$} & \multirow[b]{2}{*}{$\mathrm{n}$} & \multirow{2}{*}{\multicolumn{2}{|c|}{ Loss Functions }} & \multicolumn{5}{|c|}{ ILP } \\
\hline & & & & $\hat{D}_{1}$ & $\hat{\square}_{2}$ & $\hat{D}_{3}$ & $\hat{p}_{1}$ & $\hat{p}_{2}$ \\
\hline \multirow{18}{*}{15} & \multirow{6}{*}{25} & \multirow{2}{*}{ SELF } & $\mathrm{BE}$ & 1.50074 & 1.0113 & 0.52618 & 0.46474 & 0.30720 \\
\hline & & & PR & 0.201677 & 0.139007 & 0.059989 & 0.009709 & 0.008305 \\
\hline & & \multirow{2}{*}{ PLF } & $\mathrm{BE}$ & 1.54865 & 1.07166 & 0.59254 & 0.47333 & 0.32032 \\
\hline & & & PR & 0.121795 & 0.121661 & 0.097373 & 0.020884 & 0.026615 \\
\hline & & \multirow{2}{*}{ DLF } & $\mathrm{BE}$ & 1.60815 & 1.11961 & 0.63334 & 0.48408 & 0.33550 \\
\hline & & & PR & 0.077116 & 0.109754 & 0.158355 & 0.043828 & 0.081399 \\
\hline & \multirow{6}{*}{40} & \multirow{2}{*}{ SELF } & $\mathrm{BE}$ & 1.49739 & 1.00421 & 0.52511 & 0.48826 & 0.29331 \\
\hline & & & PR & 0.118952 & 0.091144 & 0.0375302 & 0.006323 & 0.005249 \\
\hline & & \multirow{2}{*}{ PLF } & $\mathrm{BE}$ & 1.51982 & 1.06185 & 0.56073 & 0.49484 & 0.30091 \\
\hline & & & PR & 0.074183 & 0.084245 & 0.062091 & 0.012935 & 0.017727 \\
\hline & & \multirow{2}{*}{ DLF } & $\mathrm{BE}$ & 1.55871 & 1.09042 & 0.59615 & 0.50119 & 0.31088 \\
\hline & & & PR & 0.048309 & 0.077631 & 0.107985 & 0.026403 & 0.058509 \\
\hline & \multirow{6}{*}{55} & \multirow{2}{*}{ SELF } & $\mathrm{BE}$ & 1.48179 & 0.99830 & 0.52107 & 0.48653 & 0.27762 \\
\hline & & & PR & 0.065501 & 0.065509 & 0.026486 & 0.088606 & 0.026670 \\
\hline & & \multirow{2}{*}{ PLF } & $\mathrm{BE}$ & 1.50733 & 1.04203 & 0.55675 & 0.50402 & 0.29269 \\
\hline & & & PR & 0.051611 & 0.062028 & 0.046765 & 0.026645 & 0.023686 \\
\hline & & \multirow{2}{*}{ DLF } & $\mathrm{BE}$ & 1.55419 & 1.06093 & 0.56901 & 0.50656 & 0.29809 \\
\hline & & & PR & 0.035235 & 0.059862 & 0.082028 & 0.020493 & 0.047445 \\
\hline \multirow{18}{*}{20} & \multirow{6}{*}{25} & \multirow{2}{*}{ SELF } & $\mathrm{BE}$ & 1.47373 & 1.01137 & 0.53491 & 0.46469 & 0.30656 \\
\hline & & & PR & 0.190802 & 0.137987 & 0.060765 & 0.009544 & 0.008152 \\
\hline & & \multirow{2}{*}{ PLF } & $\mathrm{BE}$ & 1.54108 & 1.05272 & 0.58151 & 0.47358 & 0.32062 \\
\hline & & & PR & 0.119934 & 0.118052 & 0.094908 & 0.020467 & 0.026116 \\
\hline & & \multirow{2}{*}{ DLF } & $\mathrm{BE}$ & 1.61174 & 1.12454 & 0.63601 & 0.48493 & 0.33430 \\
\hline & & & PR & 0.076170 & 0.1087 & 0.15734 & 0.042688 & 0.080048 \\
\hline & \multirow{6}{*}{40} & \multirow{2}{*}{ SELF } & $\mathrm{BE}$ & 1.51793 & 1.00136 & 0.52995 & 0.48844 & 0.29322 \\
\hline & & & PR & 0.121567 & 0.088951 & 0.038132 & 0.006279 & 0.005174 \\
\hline & & \multirow{2}{*}{ PLF } & $\mathrm{BE}$ & 1.53265 & 1.05414 & 0.55450 & 0.49635 & 0.30019 \\
\hline & & & PR & $\mathbf{0 . 0 7 3 9 3 7}$ & 0.082790 & 0.061166 & 0.013372 & 0.017617 \\
\hline & & \multirow{2}{*}{ DLF } & $\mathrm{BE}$ & 1.56394 & 1.09718 & 0.59098 & 0.50066 & 0.31115 \\
\hline & & & PR & 0.047889 & 0.076504 & 0.107358 & 0.025402 & 0.056618 \\
\hline & & SFI $F$ & $\mathrm{BE}$ & 1.49135 & 1.0110 & 0.52763 & 0.49673 & 0.28394 \\
\hline & & SELГ & PR & 0.082760 & 0.068240 & 0.026867 & 0.003964 & 0.003526 \\
\hline & 55 & PIF & $\mathrm{BE}$ & 1.53327 & 1.03902 & 0.54873 & 0.50575 & 0.29170 \\
\hline & JJ & PLF & PR & 0.053625 & 0.062574 & 0.045836 & 0.015091 & 0.016591 \\
\hline & & & $\mathrm{BE}$ & 1.56188 & 1.06231 & 0.56084 & 0.50672 & 0.29697 \\
\hline & & DLF & PR & 0.034834 & 0.059260 & 0.081461 & 0.018399 & 0.044454 \\
\hline
\end{tabular}


Table 17: Bayes estimates (BEs) and posterior risks (PRs) of 3-component mixture of Frechet distributions using the UP, the JP, the EP and the ILP under SELF, PLF and DLF with Crowder (1994) mixture data

\begin{tabular}{|c|c|c|c|c|c|c|c|}
\hline Prior & Loss $\mathrm{Fu}$ & ons & $\hat{\square}_{1}$ & $\hat{\square}_{2}$ & $\hat{D}_{3}$ & $\hat{p}_{1}$ & $\hat{p}_{2}$ \\
\hline \multirow{6}{*}{ UP } & \multirow{2}{*}{ SELF } & $\mathrm{BE}$ & 4.40085 & 3.52976 & 2.89294 & 0.25687 & 0.33460 \\
\hline & & PR & 0.81291 & 0.398116 & 0.21769 & 0.002437 & 0.002781 \\
\hline & \multirow{2}{*}{ PLF } & $\mathrm{BE}$ & 4.49226 & 3.58571 & 2.93033 & 0.26157 & 0.33873 \\
\hline & & PR & 0.182818 & 0.111902 & 0.074766 & 0.009401 & 0.008261 \\
\hline & \multirow{2}{*}{ DLF } & $\mathrm{BE}$ & 4.58557 & 3.64254 & 2.96819 & 0.26636 & 0.34292 \\
\hline & & PR & 0.040282 & 0.030964 & 0.025352 & 0.035618 & 0.024239 \\
\hline \multirow{6}{*}{ JP } & \multirow{2}{*}{ SELF } & $\mathrm{BE}$ & 4.22572 & 3.42494 & 2.82415 & 0.25659 & 0.33463 \\
\hline & & PR & 0.784679 & 0.387813 & 0.21316 & 0.002438 & 0.002786 \\
\hline & \multirow{2}{*}{ PLF } & $\mathrm{BE}$ & 4.31757 & 3.48109 & 2.86164 & 0.26130 & 0.33876 \\
\hline & & PR & 0.183695 & 0.112312 & 0.074980 & 0.009416 & 0.008273 \\
\hline & \multirow{2}{*}{ DLF } & $\mathrm{BE}$ & 4.41141 & 3.53817 & 2.89963 & 0.26609 & 0.34295 \\
\hline & & PR & 0.042093 & 0.032003 & 0.026030 & 0.035712 & 0.024273 \\
\hline \multirow{6}{*}{ EP } & \multirow{2}{*}{ SELF } & $\mathrm{BE}$ & 3.29247 & 2.72831 & 2.28724 & 0.26750 & 0.33895 \\
\hline & & PR & 0.137801 & 0.086027 & 0.058819 & 0.007456 & 0.006175 \\
\hline & \multirow{2}{*}{ PLF } & $\mathrm{BE}$ & 3.36137 & 2.72831 & 2.28724 & 0.26750 & 0.33895 \\
\hline & & PR & 0.137801 & 0.086027 & 0.058819 & 0.007456 & 0.006175 \\
\hline & \multirow{2}{*}{ DLF } & $\mathrm{BE}$ & 3.43171 & 2.77201 & 2.31703 & 0.27128 & 0.34207 \\
\hline & & PR & 0.040575 & 0.031283 & 0.025551 & 0.027679 & 0.018135 \\
\hline \multirow{6}{*}{ ILP } & \multirow{2}{*}{ SELF } & $\mathrm{BE}$ & 3.68196 & 3.06762 & 2.5327 & 0.25202 & 0.33045 \\
\hline & & PR & 0.589974 & 0.308466 & 0.16967 & 0.002605 & 0.003082 \\
\hline & \multirow{2}{*}{ PLF } & $\mathrm{BE}$ & 3.76123 & 3.11749 & 2.56598 & 0.25714 & 0.33508 \\
\hline & & PR & 0.158527 & 0.099745 & 0.066555 & 0.010233 & 0.009262 \\
\hline & \multirow{2}{*}{ DLF } & $\mathrm{BE}$ & 3.8422 & 3.16817 & 2.5997 & 0.26236 & 0.33978 \\
\hline & & PR & 0.041704 & 0.031739 & 0.025769 & 0.039401 & 0.027451 \\
\hline
\end{tabular}

